\title{
Transport Corrections in Nodal Diffusion Codes for HTR Modeling
}

Abderrafi M. Ougouag

Frederick N. Gleicher II

Rodolfo M. Ferrer

The INL is a

U.S. Department of Energy

National Laboratory

operated by

Battelle Energy Alliance

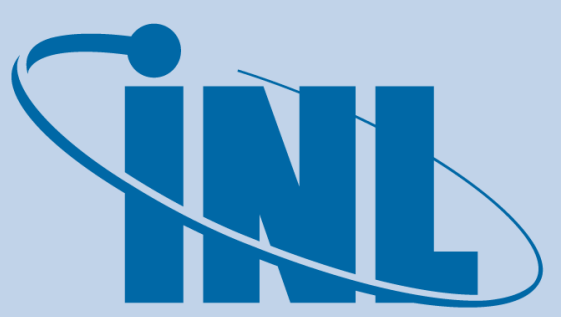

Idaho National Laboratory

August 2010

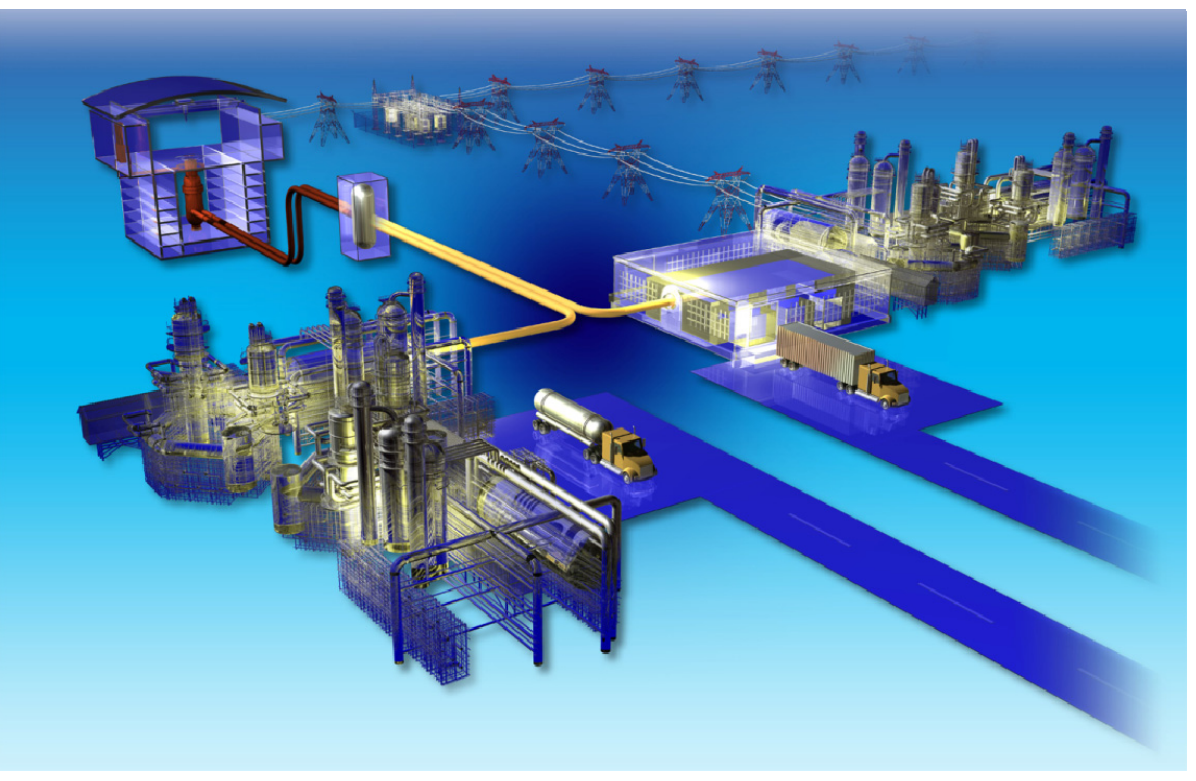




\section{DISCLAIMER}

This information was prepared as an account of work sponsored by an agency of the U.S. Government. Neither the U.S. Government nor any agency thereof, nor any of their employees, makes any warranty, expressed or implied, or assumes any legal liability or responsibility for the accuracy, completeness, or usefulness, of any information, apparatus, product, or process disclosed, or represents that its use would not infringe privately owned rights. References herein to any specific commercial product, process, or service by trade name, trade mark, manufacturer, or otherwise, does not necessarily constitute or imply its endorsement, recommendation, or favoring by the U.S. Government or any agency thereof. The views and opinions of authors expressed herein do not necessarily state or reflect those of the U.S. Government or any agency thereof. 


\title{
Transport Corrections in Nodal Diffusion Codes for HTR Modeling
}

\author{
Abderrafi M. Ougouag \\ Frederick N. Gleicher II \\ Rodolfo M. Ferrer
}

August 2010

\begin{abstract}
Idaho National Laboratory
Next Generation Nuclear Plant Project

Idaho Falls, Idaho 83415
\end{abstract}

http://www.inl.gov

Prepared for the

U.S. Department of Energy

Office of Nuclear Energy

Under DOE Idaho Operations Office

Contract DE-AC07-05ID14517 

Next Generation Nuclear Plant Project

\section{Transport Corrections in Nodal Diffusion Codes for HTR Modeling}

INL/EXT-10-19729

August 2010

Approved by:
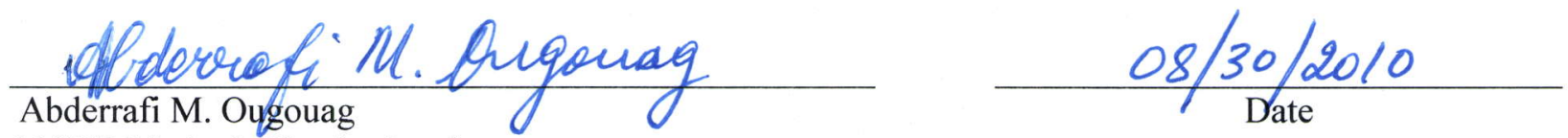

NGNP Methods Physics Lead
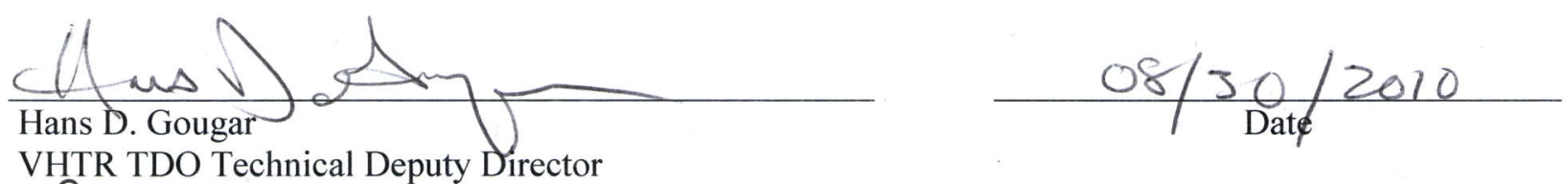

VHTR TDO Technical Deputy Director

VTR TDO Technical Deputy Director

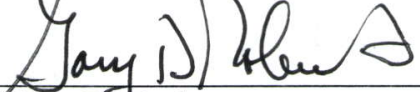

Gary D.Roberts

NGNP Quality Assurance

$\frac{08 / 30 / 2010}{\text { Date }}$ 


\begin{abstract}
The use of ordinary equivalence theory for the preparation of diffusion theory data for reactors that possess strong absorbers located in nonmultiplying regions, such as reflectors and strong localized and asymmetrically located burnable poisons, is not satisfactory. These features require transport corrections be introduced into the full core diffusion theory solutions. This report presents two such methods that are proposed to alleviate the difficulties with the use of ordinary modern equivalence and homogenization methods. The first of these methods pertains to the treatment of control rods through embedded transport theory solutions for the local transport treatment within a diffusion code. The second method pertains to an alteration of the homogenization process. In the new process, the blocks containing burnable poisons are incompletely homogenized, yielding diffusion theory data for the average modeling of the block as well as local data for the explicit treatment of the burnable poisons within the nodal diffusion solution scheme. This approach introduces two novelties: a departure from the traditional homogenization methodology and a modification to the nodal solution method that allows the explicit treatment of local heterogeneities.
\end{abstract}




\section{CONTENTS}

ABSTRACT. iv

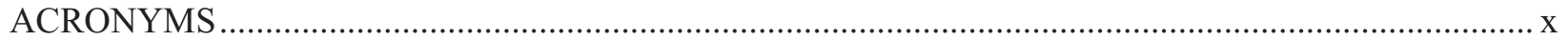

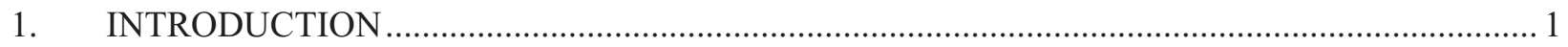

2. AN EMBEDDED TRANSPORT TREATMENT FOR CONTROL RODS................................. 2

3. HYBRID METHODOLOGY IN THE TWO-DIMENSION RADIAL AXIAL ............................. 3

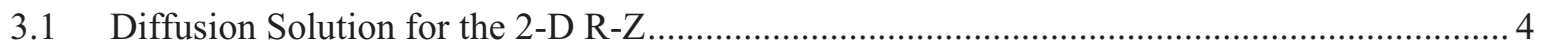

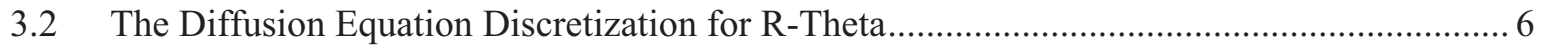

3.3 The Embedding of the Response Functions .................................................................... 8

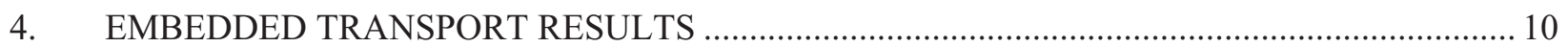

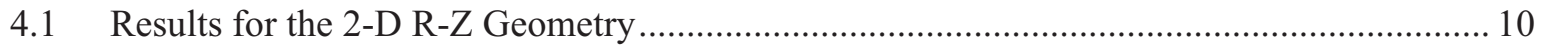

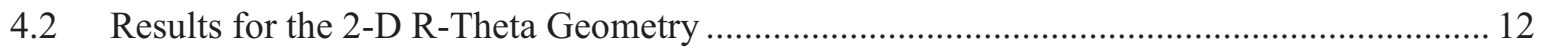

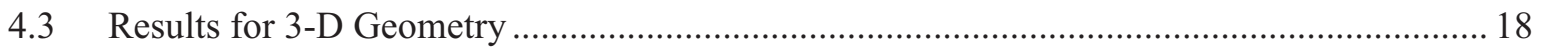

5. TRANSPORT CORRECTION METHOD FOR THE TREATMENT OF BURNABLE

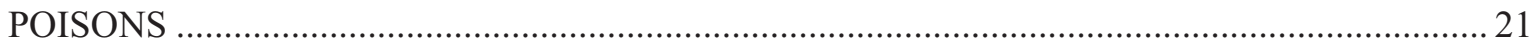

6. THEORY OF EXPLICIT TREATMENT OF STRONG HETEROGENEITIES IN

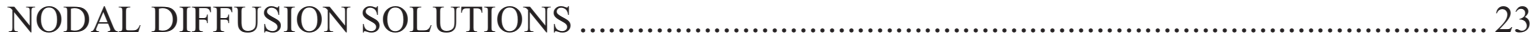

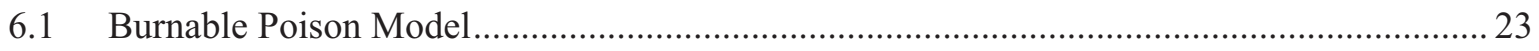

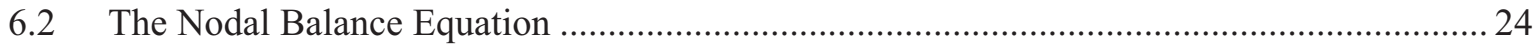

6.3 A Novel Incomplete Homogenization Method for Explicit Treatment of Strong

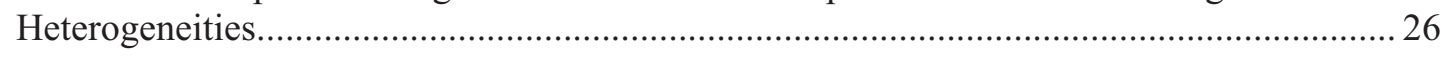

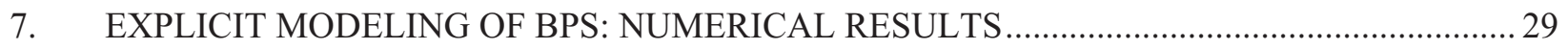

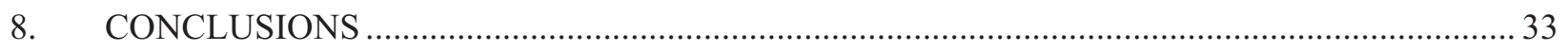

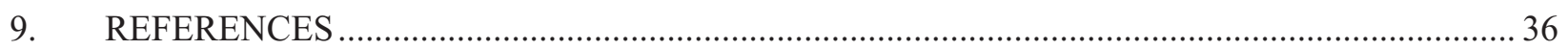

\section{FIGURES}

Figure 1. Cartoon representation of the mock PBMR core with a fully inserted control rod. 10

Figure 2. Plot of the relative error of the fast flux for the hybrid method vs. the MCNP reference solution.

Figure 3. Plot of the relative error of the thermal flux for the hybrid method vs. the MCNP reference solution.

Figure 4. Relative error the fast flux for the pure diffusion method vs. the MCNP reference solution.

Figure 5. Relative error for the thermal flux for the pure diffusion method vs. the MCNP reference solution. 
Figure 6. Diagram of the top view of the PBMR core with 24 control rods inserted.

Figure 7. Plots of the relative errors for the fast and thermal energy group scalar fluxes with 24 control rods inserted. The relative errors are obtained by comparing the scalar fluxes generated by MCNP and the fluxes generated from CYNOD Hybrid.

Figure 8. Plots of the relative error of the pure diffusion solution with 24 control rods inserted. The pure diffusion solution obtained from CYNOD is compared to an MCNP solution for the fast and thermal scalar fluxes.

Figure 9. Diagram of the reactor core with twelve control rods inserted in the reflector region.

Figure 10. Plots of the relative errors for the fast and thermal energy group scalar fluxes for 12 control rods inserted into the reflector region. The relative errors are obtained by comparing the scalar fluxes generated by MCNP and the fluxes generated from the hybrid CYNOD. 16

Figure 11. Plots of the relative error of the pure diffusion solution generated by CYNOD compared to a Monte Carlo solution generated by MCNP for the fast and thermal scalar fluxes for twelve control rods inserted into the reflector region. 16

Figure 12. Fast and thermal flux profiles for 12 randomly distributed rods.

Figure 13. Plots of the relative error for the fast and thermal fluxes for 12 randomly inserted control rods. In these plots the Hybrid method is compared to the MCNP.

Figure 14. Plots of the relative error of the fast and thermal fluxes with 12 control rods randomly inserted. The relative error was obtained by comparing pure diffusion CYNOD and MCNP. 18

Figure 15. Representation of the 3-D reactor without control rods. 19

Figure 16. Plots of the error for both the fast and thermal groups. 20

Figure 17. Simplified mini-core effective multiplication factor and power distribution without BP

Figure 18. Simplified mini-core effective multiplication factor and power distribution with center BP. 30

Figure 19. Simplified mini-core effective multiplication factor and power distribution with North BP. 30

Figure 20. Simplified mini-core effective multiplication factor and power distribution with South BP. 30

Figure 21. Simplified mini-core effective multiplication factor and power distribution with northwest BP.

Figure 22. Simplified mini-core effective multiplication factor and power distribution with West BP.

Figure 23. Simplified mini-core effective multiplication factor and power distribution with southwest BP.

Figure 24. Simplified mini-core effective multiplication factor and power distribution with southeast BP.

Figure 25. Simplified mini-core effective multiplication factor and power distribution with east BP. 
Figure 26. Simplified mini-core effective multiplication factor and power distribution with northeast BP

\section{TABLES}

Table 1. Core eigenvalues from the various methods for the fully inserted control rod........................... 10

Table 2. Critical core eigenvalues for a fully shutdown case with 24 control rods inserted..................... 13

Table 3. Eigenvalue results for 12 control rods symmetrically inserted into the outer reflector

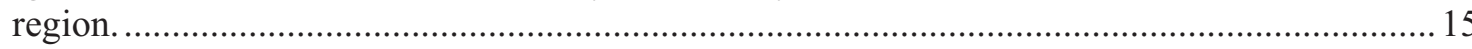

Table 4. Core eigenvalues for 12 control rods randomly distributed..................................................... 17

Table 5. Core eigenvalues for a 3-D problem without control rod elements.......................................... 19 


\section{ACRONYMS}

PBMR Pebble Bed Modular Reactor

HTR high temperature reactor

NGNP Next Generation Nuclear Plant

BP burnable poison

PBR pebble bed reactor

PMR prismatic block reactor

LWR light water reactor 


\section{Transport Corrections in Nodal Diffusion Codes for HTR Modeling}

\section{INTRODUCTION}

The cores and reflectors of high temperature reactors (HTRs) of the Next Generation Nuclear Plant (NGNP) type are dominantly diffusive media from the point of view of behavior of the neutrons and their migration between the various structures of the reactor. This means that neutron diffusion theory is sufficient for modeling most features of such reactors and transport theory may not be needed for most applications. Of course, the above statement assumes the availability of homogenized diffusion theory data. The statement is true for most situations but not all. Two features of NGNP-type HTRs require that the diffusion theory-based solution be corrected for local transport effects. These two cases are (1) the treatment of burnable poisons (BP) in prismatic block reactors and control rods embedded in nonmultiplying regions near the interface between fueled zones and said nonmultiplying zones in both pebble bed reactor (PBR) and prismatic block reactor (PMR) designs. The need for transport correction arises because diffusion theory-based solutions appear to not provide sufficient fidelity in these situations.

The reasons for the failures of diffusion theory-based solutions discussed above are multiple. However, they are not solely a failure of diffusion theory (when they actually could be ascribed to such a failure), but rather a failure of the two-step, homogenization-whole core modeling, paradigm used in conjunction with modern nodal methods. Indeed, the homogenization of the BPs and the control rods spreads the effects of the strong absorbers (BPs and control rods) throughout the homogenized region of interest. However, the regions of interest are typically large in neutronic terms (in terms of neutron mean free paths). The result is that the effect of BPs and control rods, which is in reality a localized one, is spread over large zones beyond the actual radius of influence of the strong absorber. The large zone is typically the size of a prismatic block in the case of the PMR and a spectral zone for the PBR. The BPs are typically placed in asymmetric locations, such as near some vertices of the prismatic blocks. Their effects are felt locally in their own block as well as in the adjacent blocks, but not at locations within their own block that are farther away. The homogenization process, though conserving reaction rates and leakages, spreads the reaction rates throughout the block and fails to influence neighboring blocks differently from one another (as they are in reality). The situation of the control rods is further complicated by their location in a nonmultiplying medium for which traditional homogenization methods are difficult at best and incorrect in the worst cases (when used naively). Another complication is the location of the control rods very near the fuel zone (about one mean free path from the fuel-reflector interface or the fuel-nonmultiplying block interface). At such interfaces, directional effects can be significant and diffusion theory may not apply.

The difficulties with the use of ordinary modern equivalence and homogenization methods discussed above are the subject of the correction methods presented in this report. The first of these methods pertains to the treatment of control rods through embedded transport theory solutions for the local transport treatment of control rods. The second method pertains to an alteration of the homogenization process. In the new process, the blocks containing BPs are not fully homogenized. Instead, they are only incompletely homogenized, yielding diffusion theory data for the average modeling of the block as well as local data for the explicit treatment of the BPs within the nodal diffusion solution scheme. This approach introduces two novelties: a departure from the traditional homogenization methodology and a modification to the nodal solution method that allows the explicit treatment of local heterogeneities. 


\section{AN EMBEDDED TRANSPORT TREATMENT FOR CONTROL RODS}

Accurate knowledge of the neutron radiation field as a function of space and time is essential to the correct modeling of the behavior and safety of nuclear reactors. Diffusion theory yields a good approximation to a neutron radiation field when the radiation field is weakly dependent on or independent of angle. This occurs when isotropic or weakly anisotropic scattering dominates within the medium under consideration. However, in regions with strong neutron absorbers and near nonreflective boundaries these conditions are not satisfied. For these instances, the neutron field depends highly on angle and the diffusion approximation breaks down. Yet, diffusion theory solvers that rely on nodal methods have been successfully developed for light water reactor (LWR) analysis because the transport effects of these absorbers are captured in the auxiliary process of homogenization that uses detailed single-node (assembly) transport calculations. These assembly homogenization calculations yield effective nodal diffusion parameters and discontinuity factors that allow the (mild) transport effects of LWR physics to be captured in full-core-diffusion simulations. This approach yields very accurate core-wide neutron flux distributions using a computationally efficient algorithm.

For historical reasons, application of the nodal method approach to HTRs has lagged. This is in part the consequence of an absence of commercial development or deployment of this type of reactor concept over the past two decades. The peculiarities of HTRs (long mean free path, cylindrical or hexagonal geometry, and moving fuel in the case of the pebble bed reactor) are not adequately treated by existing LWR analysis tools. In particular the angular effects on the neutron field by control rods in HTRs are not adequately captured through homogenization techniques like they are in LWRs.

The modeling of control rods in HTRs has been approached in various ways with varying degrees of success. In traditional whole-core simulation, the coupling between nodes is achieved using coupling coefficients derived from the diffusion theory parameters generated in an assembly transport calculation. Such calculations are difficult to perform for reflector nodes because of the lack of a neutron source. Usually a small amount of fuel is artificially added to the reflector composition that surrounds the control rod to generate the neutron flux, which in reality streams in from adjacent nodes. This approach yields spatial and spectral errors in the nodal flux. A more successful approach is the Method of Equivalent Cross Sections, ${ }^{1}$ in which a fine mesh transport solution is obtained for the heterogeneous region with the control rod, and the reaction rates are calculated for that region. This heterogeneous region is called a super cell. Calculated leakage rates of the fine mesh transport are matched to the calculated leakage rates of the neighboring nodes where the neighboring nodes are treated with diffusion. An equivalent average flux for the super cell is computed based on the average cell flux and edge-cell flux values of the neighboring diffusion treated nodes. The equivalent cross sections are then obtained from the super-cell average flux and the reaction rates. This procedure is done in the Method of Equivalent Cross Sections through analyst intervention - careful specification of the thickness of the super cell, such that the leakage rates of the transport solution are equivalent to those of an auxiliary diffusion solution. Transport and diffusion leakage rates must match at the edges of the super cell. Since the control rod is often at the center of the super cell, there are restrictions on the location and size of the absorber node that can be inconsistent with the nodalization of the rest of the model and lead to some analytical uncertainty. Overall, this method requires considerable engineering judgment and is thus sensitive to core design and prone to uncertainty.

Another approach for the treatment of control rods is the use of modified boundary conditions with response functions, which was first explored by Fen et al. ${ }^{2}$ This approach generates response functions from the assembly calculation which are used as modified boundary conditions in the whole core diffusion calculation. Response functions relate the normalized partial current impinging on one face of a node to the currents leaving that face and the other, remaining, faces. Like the normal diffusion parameters, response functions can be generated from assembly transport calculations, but they do not require an internal source and they directly account for the spatial asymmetry of internodal currents. The response function method avoids having to choose possibly thick regions to capture equivalent leakages 
when control rods are present. However, the Fen method has only been only applied to a control rod region internal to the core and has not been applied to the reactor boundary. Should a response region need to be changed or extended, the analyst must rerun the entire response calculation to obtain new boundary conditions.

To obtain a method that does not require significant analyst intervention and that can be embedded into a highly accurate nodal diffusion solver, an embedded response nodal formulation was developed in 1-D geometry. ${ }^{3}$ The method differs significantly from the second method discussed above by incorporating the response functions directly within the nodal formulation as an embedded scheme; the response functions are used to generate coupling coefficients morphologically identical to the diffusiontheory-based coefficients they replace. This method also avoids having to rerun each calculation if the response region changes, since each new response region can be incorporated seamlessly. However, the response functions in this method do not capture the spatial and angular variation across the surface of a node. These variations result when the neutrons enter the response node and encounter objects or nonsymmetric material regions or when neutrons travel from the response node surface through the response node to the adjacent surface. To account for these effects across the response node, higher order space and angle response moments are calculated or the set of response functions for adjacent response (concurrent) nodes are calculated together, becoming tightly coupled to each other. ${ }^{4}$ If one wants to directly embed the response functions into an existing nodal solver, the higher order response function moments in space and angle makes the embedding difficult. The difficulty results because the system of equations is nonsymmetric and the extra spatial and angular moments must be directly incorporated into the existing matrix for the nodal net currents or the average nodal flux. On the other hand, the tightly coupled concurrent response nodes introduce the difficulty replacing nodes. If the control rod position is slightly changed, an entire new set of response functions must be calculated even though physical morphology of the other response nodes does not change.

In this report, a new method for the treatment of control rods is developed in 2-D (R-Z and R-Theta) geometry. The control rod region is modeled with response functions, but differs significantly from both Fen's method and the one discussed above. In this new method, the response function region and diffusion regions are coupled together through the interaction of partial currents at the adjoined node boundaries, much like Fen's response method. However, unlike Fen's method the response region in the new method is divided into nodes, and the higher incoming current moments in space and angle are calculated to adjoin response nodes. This allows for the calculation of accurate nodal scalar fluxes and net currents for the designated response nodes, and the response nodes can be swapped without affecting the other neighboring nodes.

Moreover, the solution for the response region is directly embedded into the nodal solver's source iteration, but the response region is swept separately to avoid difficulty with the equations of a direct embedding into the matrix for the average nodal scalar flux. Thus the new method is a seamless embedding that avoids the difficulties of incorporating the response functions directly into a symmetric nodal diffusion solver.

The rest of this report is laid out as follows: Section 3 presents the new hybrid methodology, Section 4 gives the results of embedded transport, Section 5 presents the transport correction method for the treatment of BPs, Section 6 presents the theory of explicit treatment of strong heterogeneities in nodal diffusion solutions, Section 7 presents the numerical results of explicit modeling of BPs and Section 8 is the conclusion.

\section{HYBRID METHODOLOGY IN THE TWO-DIMENSION RADIAL AXIAL}

In this section the hybrid method is developed in the 2-D R-Z and R-Theta geometries. The nodal scalar flux is calculated by applying a highly-accurate coarse mesh finite difference method to the 
homogenized fuel and inner reflector regions and applying a response function moment method to the surrounding nonmultiplying reflecting nodes. The critical core eigenvalue is calculated by the two-level iteration processes with inner and outer iterations. This two-level iteration algorithm is well known and the details are covered in literature. ${ }^{5}$ This section summarizes the 2-D R-Z and R-Theta diffusion methodologies, then discusses the embedded response function methodology coupled to the both diffusion methods.

\subsection{Diffusion Solution for the 2-D R-Z}

To obtain an accurate numerical solution for the scalar flux for an azimuthally symmetric reactor, the hybrid method must be a robust and accurate numerical diffusion solution. The multigroup k-eigenvalue neutron diffusion equation for an azimuthally symmetric cylinder is

$$
-\frac{1}{r} \frac{\partial}{\partial r}\left(r D_{r, g} \frac{\partial \phi_{g}(r, z)}{\partial r}\right)-\frac{\partial}{\partial z}\left(D_{z, g} \frac{\partial \phi_{g}(r, z)}{\partial z}\right)+\Sigma_{R, g} \phi_{g}(r, z)=S_{g}(r, z) .
$$

In Equation 1 the radial and axial multigroup diffusion coefficients are $D_{r, g}(r, z)$ and $D_{z, g}(r, z)$, the homogenized multigroup removal cross section is $\Sigma_{R, g}$, and the energy group scalar flux is $\phi_{g}(r, z)$. The source for group $\mathrm{g}$ for the right hand side of Equation 1 is

$$
S_{g}(r, z)=\sum_{g^{\prime} \neq g} \Sigma_{s, g^{\prime} \rightarrow g} \phi_{g}(r, z)+\frac{\chi_{g}}{k_{\text {eff }}} \sum_{g^{\prime}=1}^{N_{g}} v_{f, g} \Sigma_{f, g} \phi_{g}(r, z),
$$

where $\Sigma_{s, g^{\prime} \rightarrow g}$ is the energy group to group scattering cross section, $\chi_{g}$ is the fission spectrum for group $\mathrm{g}, k_{\text {eff }}$ is core eigenvalue, $v_{f, g}$ is the average number neutrons produced per fission event, and $\Sigma_{f, g}$ is the fission cross section. The domain is the cylinder $\{\Omega(\mathrm{r}, \mathrm{z}): 0 \leq \mathrm{r} \leq \mathrm{R} ; 0 \leq \mathrm{z} \leq \mathrm{L}\}$. The boundary conditions for Equation 1 are

- $J^{g}(0, z)=0$

- $\quad \frac{1}{4} \phi^{g}(R, z)-\frac{1}{2} J^{g}(R, z)=J^{i n, R}$

- $\frac{1}{4} \phi^{g}(r, 0)+\frac{1}{2} J^{g}(r, 0)=0$

- $\frac{1}{4} \phi^{g}(r, L)-\frac{1}{2} J^{g}(r, L)=0$.

The first boundary condition specifies radial symmetry at the radial cylinder center and the second boundary condition specifies an incoming current at the radial cylinder edge. The third and fourth boundary conditions specify zero incoming current on the top and bottom of the cylinder. To obtain a numerical solution to Equation 1, the domain is subdivided into subdomains called nodes. For each node the balance equation for each energy group is spatially integrated and divided by the node volume to obtain

$$
\frac{2 r_{b}}{r_{b}^{2}-r_{a}^{2}} \bar{J}_{i, k, g}^{r_{b}}-\frac{2 r_{a}}{r_{b}^{2}-r_{a}^{2}} \bar{J}_{i, k, g}^{r_{a}}+\frac{1}{2 a_{z}} \bar{J}_{i, k, g}^{a_{z}}-\frac{1}{2 a_{z}} \bar{J}_{i, k, g}^{-a_{z}}+\Sigma_{R, i, k, g} \bar{\phi}_{i, k, g}=\bar{Q}_{i, k, g}
$$

Equation 2 is the nodal balance equation for a 2-D r-z node that has been spatially homogenized. The node domain is the cylindrical ring $\left\{\Omega(r, z): r_{a} \leq r \leq r_{b},-a_{z} \leq z \leq a_{z}\right\}$. The radial index for the node is $i$ and 
the axial index for the node is $\mathrm{k}$. The net currents $\bar{J}_{i, k, g}^{r_{b}}$ and $\bar{J}_{i, k, g}^{r_{a}}$ are the axially averaged net currents at the respective outer and inner radial boundaries, and the net currents $\bar{J}_{i, k, g}^{-a_{z}}$ and $\bar{J}_{i, k, g}^{a_{z}}$ are the net currents averaged over the radial direction at the respective bottom and top node boundaries. The average node flux is $\bar{\phi}_{i, j, g}$, and $\bar{Q}_{i, j, g}$ is the averaged source for the node. To obtain a linear system of equations for the node average flux, the surface net currents in Equation 2 are expressed in terms of the node average fluxes. A highly accurate semi-analytic technique that obtains equations for the net current in terms of the average nodal scalar flux is the Analytical Nodal Green's function method ${ }^{6}$ or its ILLICO equivalent. ${ }^{7}$ The Analytical Nodal Green's Function method gives expressions for the scalar flux in terms of the average net currents on a node boundary in the following way. First for each spatial direction, a transverse direction is chosen and a 2-D diffusion equation is spatially integrated over the transverse direction for each node. The transverse currents evaluated at the node boundaries are known as the transverse leakage terms, and are combined into the node source term. The resulting 1-D diffusion equations are solved by a Green's Function technique, and analytic expressions for the scalar flux are obtained. The analytical expressions for the scalar flux derived for both the axial and radial directions are

$$
\phi_{g}(z)=G\left(-a_{z}, z\right) J_{i, k}^{g,-a_{z}}-G\left(a_{z}, z\right) J_{i, k}^{g, a_{z}}+\int_{-a_{z}}^{a_{z}} G\left(z, z^{\prime}\right) S\left(z^{\prime}\right) d z
$$

and

$$
\phi_{g}(r)=r_{a} G\left(r_{a}, r\right) J_{i, k}^{g, r_{a}}-r_{b} G\left(r_{b}, r\right) J_{i, k}^{g, r_{b}}+\int_{r_{a}}^{r_{b}} r^{\prime} G\left(r, r^{\prime}\right) S\left(r^{\prime}\right) d r^{\prime}
$$

Where

$$
\phi_{g}(r, z)=\phi_{g}(r)+\phi_{g}(z)-\bar{\phi}_{r, z, g},
$$

the functions $G\left(z, z^{\prime}\right)$ and $G\left(r, r^{\prime}\right)$ are the axial and radial Green's Functions, respectively, and the terms $S\left(r^{\prime}\right)$ and $S\left(z^{\prime}\right)$ are the radial and axial source terms that contain the up-scatter, down-scatter, fission source, and transverse leakage terms. The term $\bar{\phi}_{r, z}$ in Equation 5 is the average scalar flux. The scalar flux at the node surfaces are obtained from Equations 3 and 4 by evaluating Equation 4 at the node radial boundaries and Equation 3 at the node axial boundaries. A continuity (or discontinuity) condition is introduced at the surfaces of a node to relate the nodal surface scalar fluxes to the surface scalar fluxes of neighboring nodes. ${ }^{8,9}$ The continuity condition for the bottom axial node surface is

$$
f_{i, k-1, g}^{a_{z}} \phi_{i, k-1, g}^{a_{z}}=f_{i, k, g}^{-a_{z}} \phi_{i, k, g}^{-a_{z}}
$$

where $f_{i, k-1, g}^{a_{z}}$ and $f_{i, k, g}^{-a_{z}}$ are discontinuity factors and the fluxes $\phi_{i, k-1, g}^{a_{z}}$ and $\phi_{i, k, g}^{-a_{z}}$ are the surface fluxes at the bottom of node (i, k) and the top of node (i, k-1). Expressions for the other scalar flux continuity (or discontinuity) condition are written down for the other node surfaces. The result is a set of equations that are manipulated to obtain the net current at the node boundary. These equations contain terms with the node average scalar flux. For example, the net current at the bottom node boundary is $J_{i, k, g}^{a_{z}}=D_{i, k, g}^{-a_{z}} \bar{\phi}_{i, k, g}-D_{i, k-k, g}^{a_{z}} \bar{\phi}_{i, k-1, g}+E_{i, k, g} \bar{S}_{i, k, g}-E_{i, k-1, g} \bar{S}_{i, k-1, g}-\frac{D_{i, k, g}^{-a_{z}}}{C_{k}^{f}} \int_{-a_{z}}^{a_{z}} G\left(-a_{z}, z^{\prime}\right) S\left(-a_{z}, z^{\prime}\right) d z+\frac{D_{i, k-1, g}^{a_{z}}}{C_{k-1}^{f}} \int_{-a_{z}, k-1}^{a_{z, k-1}} G_{k-1}\left(a_{z, k-1}, z^{\prime}\right) S\left(a_{z, k-1}, z^{\prime}\right) d z$

In Equation 7, $\bar{\phi}_{i, k, g}$ and $\bar{\phi}_{i, k-1, g}$ are the node average fluxes for the current and bottom neighboring node. $\bar{S}_{i, k, g}$ and $\bar{S}_{i, k-1, g}$ are the node sources and $D_{i, k, g}^{-a_{z}}, E_{i, k, g}$, and $C_{k}^{f}$ are coefficients resulting from 
the algebra. The last integral in Equation 7 has indices that indicate the integral is evaluated for the node below the current node. Another example is the net current at the inner radial boundary which is given by

$$
J_{i, k, g}^{r_{a}}=D_{i, k, g}^{r_{a}} \bar{\phi}_{i, k, g}-D_{i-1, k, g}^{b_{b}} \bar{\phi}_{i-1, k, g}-H_{i, k, g}^{r_{a}} \bar{S}_{i, k, g}+H_{i-1, k, g}^{r_{b}} \bar{S}_{i-1, k, g}-\frac{1}{\Lambda_{i, j, k, g}} \int_{r_{a}}^{r_{b}} r G\left(r_{a}, r_{0}\right) S\left(r_{a}, r_{0}\right) d r_{0}+\frac{1}{\Lambda_{i-1, j, k, g}} \int_{r_{a, i-1}}^{r_{b, i-1}} r G_{i-1}\left(r_{b, i-1}, r_{0}\right) S\left(r_{b, i-1}, r_{0}\right) d r_{0}
$$

The coefficients $H_{i, k, g}^{r_{a}}, H_{i-1, k, g}^{r_{b}}, \Lambda_{i, k, g}$, and $\Lambda_{i-1, k, g}$ in Equation 8 result from algebra, and the last integral is evaluated over the inner node adjacent to the current node. Similar expressions are derived for the outer radial and the top axial boundary average net current, and the resulting equations for the average net current expressed in Equations 7 and 8 are exact. However, the integral terms in Equations 7 and 8 are difficult to evaluate exactly analytically and are therefore approximated with a truncated Legendre polynomial expansion. The approximation of the integrals in Equation 7 gives

$J_{i, k, g}^{-a_{z}}=-D_{i, k, g}^{-a_{z}} \bar{\phi}_{i, k, g}+D_{i, k-1, g}^{a_{z}} \bar{\phi}_{i, k-1, g}+E_{i, k, g} \bar{S}_{i, k, g}-E_{i, k-1, g} \bar{S}_{i, k-1, g}-\frac{D_{i, k, g}^{-a_{z}}}{C_{k}^{f}} \sum_{m=1}^{M} G_{i, k, g}^{-m} S_{i, k, g}^{-m}+\frac{D_{i, k-1, g}^{a_{z}}}{C_{k-1}^{f}} \sum_{m=1}^{M} G_{i, k, g}^{+, m} S_{i, k, g}^{+, m}$.

The terms $G_{i, k, g}^{-, m}$ and $G_{i, k, g}^{+, m}$ in Equation 9 are the Legendre expansion moments of the Green's

Function with an evaluation at the top and bottom node boundaries, and the terms $S_{i, k, g}^{-, m}$ and $S_{i, k, g}^{+, m}$ are the Legendre source expansion moments. Similar expressions for the node average net current are obtained for the inner and outer radial node boundaries and the top node boundary. The equations for the axial net currents are substituted into Equation 2, and the average net currents on the radial boundaries are combined into the source. The node average fluxes are then algebraically manipulated to form a three stripe matrix row which is given as

$A_{k+1}^{g, R} \bar{\phi}_{i, k+1, g}+\left(A_{k+1}^{g, L}+A_{k-1}^{g, R}\right) \bar{\phi}_{i, k, g}+A_{k-1}^{g, L} \bar{\phi}_{i, k-1, g}=\bar{S}_{i, k, g}$.

The algebraic combined terms in Equation 10 are expressed and the source $\bar{S}_{i, k, g}$ contains all sources, including neutrons that down-scatter and neutrons that are produced from fission. The source term also contains the transverse leakage terms from the inner and outer radial sides. Tri-diagonal systems of equations for the node average scalar flux in the axial direction are formed for each radial mesh domain, and tri-diagonal matrix systems for the node average scalar flux in the radial direction are formed for each axial mesh level. The tri-diagonal systems can be solved if the right hand side term is known. However, since these terms are unknown, the node edge net currents, the up-scatter and down-scatter sources, and the fission source are initially guessed.

The axial tri-diagonal systems of equations along the radial direction are solved to obtain the node average scalar flux. These are the sweeps in the axial direction. Then the axial transverse leakage terms are updated and the radial tri-diagonal systems of equations for the node average scalar flux are solved. The radial transverse leakage terms are then updated and the axial direction is swept again. In this way the node sources, scalar fluxes, and edge currents are iterated upon until they converge. This algorithm for obtaining the average node scalar fluxes and sources is known to be efficient, yielding the correct solution for the average nodal flux. ${ }^{7}$ The source iterations discussed above are the outer iteration.

\subsection{The Diffusion Equation Discretization for R-Theta}

To obtain an accurate numerical solution for the scalar flux for an infinite cylindrical reactor, the hybrid method must have a robust and accurate numerical diffusion solution. The multigroup keigenvalue neutron diffusion equation for an infinitely tall cylinder is 


$$
-\frac{1}{r} \frac{\partial}{\partial r}\left(r D_{r, g} \frac{\partial \phi_{g}(r, \theta)}{\partial r}\right)-\frac{1}{r^{2}} \frac{\partial}{\partial \theta}\left(D_{\theta, g} \frac{\partial \phi_{g}(r, \theta)}{\partial \theta}\right)+\Sigma_{R, g} \phi_{g}(r, \theta)=S_{g}(r, \theta) .
$$

The radial and axial multigroup diffusion coefficients in Equation 11 are $D_{r, g}(r, \theta)$ and $D_{\theta, g}(r, \theta)$, the multigroup removal cross section is $\Sigma_{R, g}$, and the energy group scalar flux is $\phi_{g}(r, \theta)$. The source for group $\mathrm{g}$ for the right hand side of Equation 11 is

$$
S_{g}(r, \theta)=\sum_{g^{\prime} \neq g} \Sigma_{s, g^{\prime} \rightarrow g} \phi_{g}(r, \theta)+\frac{\chi_{g}}{k_{\text {eff }}} \sum_{g^{\prime}=1}^{N_{g}} v_{f, g} \Sigma_{f, g} \phi_{g}(r, \theta)
$$

where $\Sigma_{s, g^{\prime} \rightarrow g}$ is the energy group to group scattering cross section, $\chi_{g}$ is the fission spectrum for group $\mathrm{g}, k_{\text {eff }}$ is the core eigenvalue, $v_{f, g}$ is the average number neutrons produced per fission event, and $\Sigma_{f, g}$ is the fission cross section. The domain is the infinite cylinder $\{\Omega(r, \theta): 0 \leq \mathrm{r} \leq \mathrm{R} ; 0 \leq \theta \leq 2 \pi\}$, and the boundary conditions for Equation 11 are:

- $J^{g}(0, \theta)=0$

- $\quad \frac{1}{4} \phi^{g}(R, \theta)-\frac{1}{2} J^{g}(R, \theta)=J^{i n, R}$

- $\quad J^{g}(r, 0)=J^{g}(r, 2 \pi)$

- $\phi^{g}(r, 0)=\phi^{g}(r, 2 \pi)$.

The first boundary condition specifies radial symmetry at the radial cylinder center, and the second boundary condition specifies an incoming current at the radial cylinder edge. The third and fourth boundary conditions specify azimuthal continuity of the scalar flux and current. To obtain a numerical solution to Equation 11 the domain is subdivided into subdomains called nodes. The node balance equation is derived by spatially integrating the balance equation over each node and dividing by the node volume. The node balance equation for a spatially homogenized node is

$$
\frac{2 r_{b}}{r_{b}^{2}-r_{a}^{2}} \bar{J}_{i, k, g}^{r_{b}}-\frac{2 r_{a}}{r_{b}^{2}-r_{a}^{2}} \bar{J}_{i, k, g}^{r_{a}}+\frac{1}{\alpha_{0}\left(r_{b}+r_{a}\right)} \bar{J}_{i, j, g}^{\alpha_{0}}-\frac{1}{\alpha_{0}\left(r_{b}+r_{a}\right)} \bar{J}_{i, j, g}^{-\alpha_{0}}+\Sigma_{R, i, j, g} \bar{\phi}_{i, j, g}=\bar{Q}_{i, j, g}
$$

The domain for the node is $\left\{\Omega(r, \theta): r_{a} \leq r \leq r_{b},-\alpha_{0} \leq \theta \leq \alpha_{0}\right\}$ where the radial index for the node is $i$ and, the azimuthal index for the node is $\mathrm{j}$. The net currents $\bar{J}_{i, j, g}^{r_{b}}$ and $\bar{J}_{i, j, g}^{r_{a}}$ are the azimuthally averaged net currents at the respective outer and inner radial boundaries, and the net currents $\bar{J}_{i, j, g}^{-\alpha_{0}}$ and $\bar{J}_{i, j, g}^{\alpha_{0}}$ are the net currents averaged over the radial direction at the azimuthal boundary sides. The average node flux is $\bar{\phi}_{i, j, g}$, and $\bar{Q}_{i, j, g}$ is the averaged source for the node. A linear system of equations for the node average flux can be obtained from Equation 12 if a set of equations for the node edge average net currents can be expressed in terms of the node average fluxes. The average net current equations for the inner and outer radial boundary are obtained from the Analytical Nodal Green's Function method. This method expresses the average edge net currents in terms of two adjoined node average fluxes and a set of nodal sources. Equation 8 is an example of the inner radius net current obtained from the Analytical Nodal Green's Function method manipulation, and again the integrals are expanded with Legendre polynomials. Furthermore, the average net currents at the azimuthal node sides are obtained from a finite difference derivation. 
The derivation of the finite difference equations are well documented ${ }^{10,11}$ and are not repeated here for this report. The equation azimuthal current for side $-\alpha_{0}$ is

$$
J_{i, j, g}^{-\alpha_{0}}=-D_{i, j, g}^{-\alpha_{0}, \theta}\left(\bar{\phi}_{i, j, g}-\bar{\phi}_{i, j-1, g}\right)
$$

and the equation for the side $\alpha_{0}$ is

$$
J_{i, j, g}^{\alpha_{0}}=-D_{i, j, g}^{\alpha_{0}, \theta}\left(\bar{\phi}_{i, j+1, g}-\bar{\phi}_{i, j, g}\right) \text {. }
$$

The coefficients $D_{i, j, g}^{-\alpha_{0}, \theta}$ and $D_{i, j, g}^{\alpha_{0}, \theta}$ result from algebraic manipulation of the finite difference method, and $\bar{\phi}_{i, j, g}$ is the average nodal scalar flux.

In addition, to obtain equations for the average nodal flux the equations for the inner and outer radial net currents are substituted into Equation 12, and the net currents on the azimuthal boundaries are combined into the source. The node average fluxes are then algebraically manipulated to form a threestripe matrix row for the radial direction. Tri-diagonal systems of equations for the node average scalar flux in the radial direction are formed for each azimuthal node domain. Similar matrix systems are formed for the node average scalar flux in the azimuthal direction for each radial node domain. However, because of the continuation boundary conditions on the azimuthal flux and current, the equations for the node average flux in the azimuthal direction are not strictly tri-diagonal.

The tri-diagonal systems in the radial direction can be solved if the right hand side term is known. However, since these terms are unknown, the node edge net currents, up-scatter and down-scatter sources, and fission source are initially guessed.

The radial tri-diagonal systems of equations are solved to obtain the scalar flux along the counter clockwise azimuthal direction. This is a sweep in the radial direction. Then the transverse leakage terms in the radial direction are updated and the matrix systems of equations in the azimuthal direction are solved to obtain the node average scalar flux. The transverse leakage terms in the azimuthal direction are then updated and the radial direction is swept again. In this way the node sources, scalar fluxes, and edge currents are iterated upon until they converge. This algorithm for obtaining the average node scalar fluxes and sources is known to be efficient, yielding the correct solution for the average nodal flux. ${ }^{7}$ The source iterations discussed above are the outer iteration.

\subsection{The Embedding of the Response Functions}

The designated transport region is subdivided into nodes much like the diffusion region, but unlike the diffusion region, the numerical solution is computed with response functions. The quantity $Z_{i, k}^{g, p^{\prime}, X}$ in energy group g, for response moment p', averaged over the volume or area of interest (denoted by X which stands for the volume of the node for the node (i,k) or for the node surface $\mathrm{f}$ ) is formulated by means of the response functions according to ${ }^{12,13}$

$$
Z_{i, k}^{g, p^{\prime}, X}=\sum_{p} \sum_{g^{\prime}=1}^{N_{g}} \sum_{f} R_{\substack{g^{\prime} \rightarrow g \\ p \rightarrow p^{\prime}}}^{Z_{i, p^{\prime}, X}} J_{i, k}^{i n, g^{\prime}, p, f}
$$

where $J_{i, k}^{i n, g, p, f}$ is the partial incoming current moment from the surface $f$ into node (i,k), and $R_{g^{\prime} \rightarrow g, f \rightarrow X}^{Z_{i, p^{\prime}, X}}$ represents the partial current response functions for the given integral property $Z_{i, k}^{g, p^{\prime}, X}$. Typical quantities of interest are the average response nodal flux $\bar{\phi}_{i, k, g}$, the heterogeneous surface fluxes $\phi_{i, k, g}^{f}$, and the net current moments $J_{i, k}^{n e t, g, p^{\prime}, f}$. The indices (i,k) are used for either 2-D R-Z or R-Theta pairing. The diffusion region supplies the incoming currents to the adjoined transport nodes. The partial currents and other quantities of interest are then obtained either by a sweeping method or a direct solve. The outgoing 
currents at the adjoined diffusion/response boundary from the response region become a new set of inputs to the diffusion region and are incorporated into the nodal source of the adjoined diffusion nodes. The nodal sources to the diffusion region are then updated, and new average nodal scalar flux values are obtained. In this way, the response and diffusion regions are iterated upon until the average nodal scalar flux in both regions are converged to a specified tolerance. 


\section{EMBEDDED TRANSPORT RESULTS \\ 4.1 Results for the 2-D R-Z Geometry}

To demonstrate the capturing of the anisotropic effects of the control rods and boundary in the 2-D R$\mathrm{Z}$ geometry, a simple mock pebble bed modular reactor (PBMR) core with fully inserted control rods was modeled. The reactor core is $400 \mathrm{~cm}$ high with a total radius of $235 \mathrm{~cm}$. The fuel region is $350 \mathrm{~cm}$ high and $85 \mathrm{~cm}$ wide, starting by the inner reflector. The inner reflector is $100 \mathrm{~cm}$ wide starting at the radial center, and the outer reflector, which contains a fully inserted control rod, is $50 \mathrm{~cm}$ wide. The control rod is near the reactor core. A simple diagram of the reactor is shown in Figure 1.

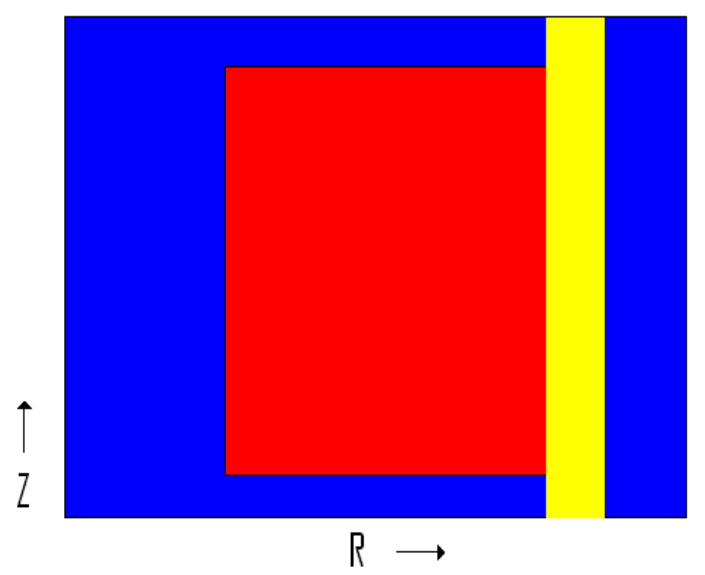

Figure 1. Cartoon representation of the mock PBMR core with a fully inserted control rod.

The hybrid method was examined with three different couplings to the incoming current of the designated diffusion region. In all cases the diffusion region was designated to be the inner reflector and the core region, and the transport region was designated to be the control rod and outer reflector. For the first coupling the incoming currents to the diffusion region are obtained from albedos given by a reference solution, and in the second coupling the incoming currents are obtained from an embedded code that interacted with a segmented response function over the outer rod and reflector region. The second coupling is very close to the method implemented by Fen et al. ${ }^{2}$ The third coupling is the hybrid method with the embedded individual response nodes. The results of the core eigenvalue are shown in Table 1.

Table 1. Core eigenvalues from the various methods for the fully inserted control rod.

\begin{tabular}{|l|c|}
\hline \multicolumn{1}{|c|}{ Case } & Core Eigenvalue $\left(\mathbf{K}_{\text {eff }}\right)$ \\
\hline Reference & 0.90243 \\
\hline Pure Diffusion & 0.90488 \\
\hline Albedos & 0.90183 \\
\hline Embedded Response Code (Fen et. al. method) & 0.90181 \\
\hline Embedded Response Code & 0.90170 \\
\hline
\end{tabular}

A reference solution was obtained from a Monte Carlo N-Particle (MCNP) transport code model. The Fen method and the albedos from the reference solution produce nearly the same critical core eigenvalue. In both cases the difference between the reference eigenvalue and the calculated solution from the Fen et al. hybrid is $0.06 \%$. The hybrid with the individual response nodes generates a slightly higher error of $0.08 \%$, and the pure diffusion result produces a difference of $0.27 \%$. The small increase in error between the hybrid with the individual response nodes and the Fen and albedo methods are expected. A small 
amount of extra error is generated by subdividing the designated region into response nodes, each of which have some small truncation error in the response representation. The albedo and the Fen et al. methods do not have truncation error, but the entire region must be recalculated if perturbation in the control position or composition occurs for these two methods.

Furthermore, the nodal fluxes obtained from the hybrid method are compared against the fluxes obtained from the reference solution. As shown in Figures 2 and 3, the absolute error of the average nodal flux shows good agreement with the reference solution. The maximum error for the fast group in the embedded response function case occurs around the control rod near the boundary and is less than $4.0 \%$, while The maximum error for the thermal group is less than $1.8 \%$. The error in power region of the core is shown to be less than $1 \%$. These results should be contrasted with those obtained from pure nodal diffusion shown in Figures 4 and 5. The relative errors for the fast energy group in the diffusion solution are less than $4.0 \%$, but the relative errors for the thermal energy group in the diffusion solution are up to $36.0 \%$. Also, the fast flux shows errors greater than $1 \%$ in the power region of the core. These effects are caused by the angular effects of the control rod, which causes diffusion to break down.

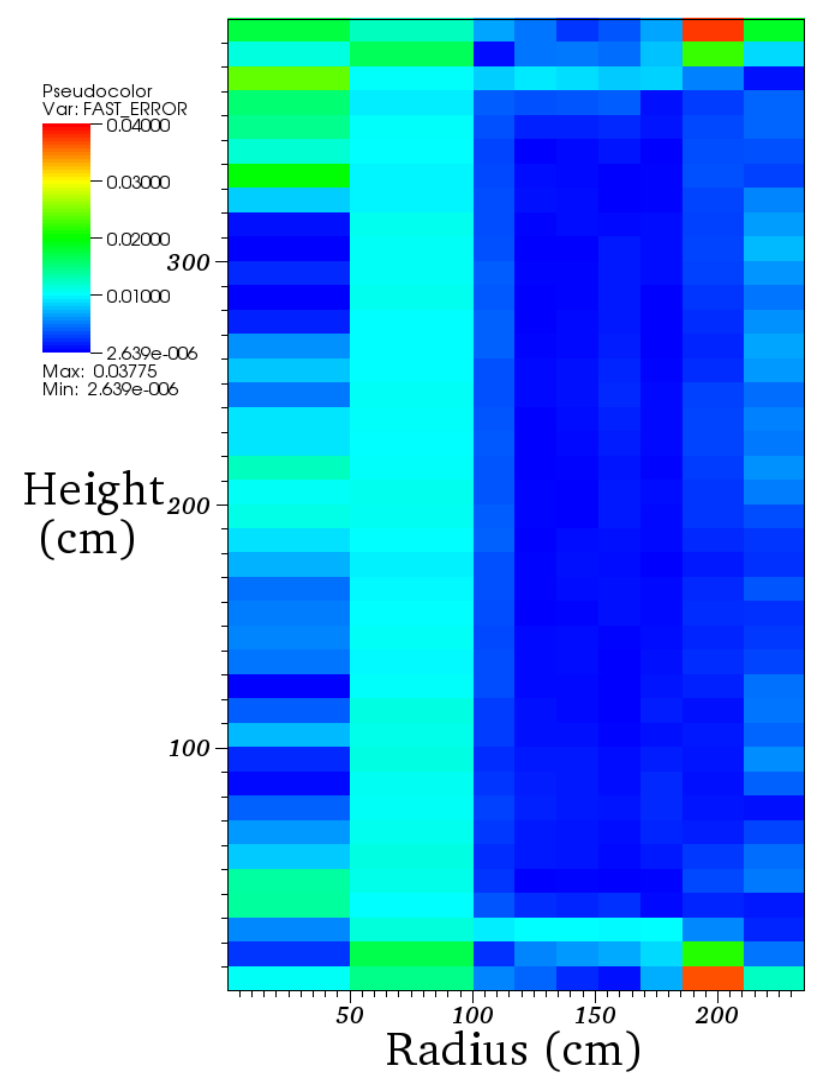

Figure 2. Plot of the relative error of the fast flux for the hybrid method vs. the MCNP reference solution.

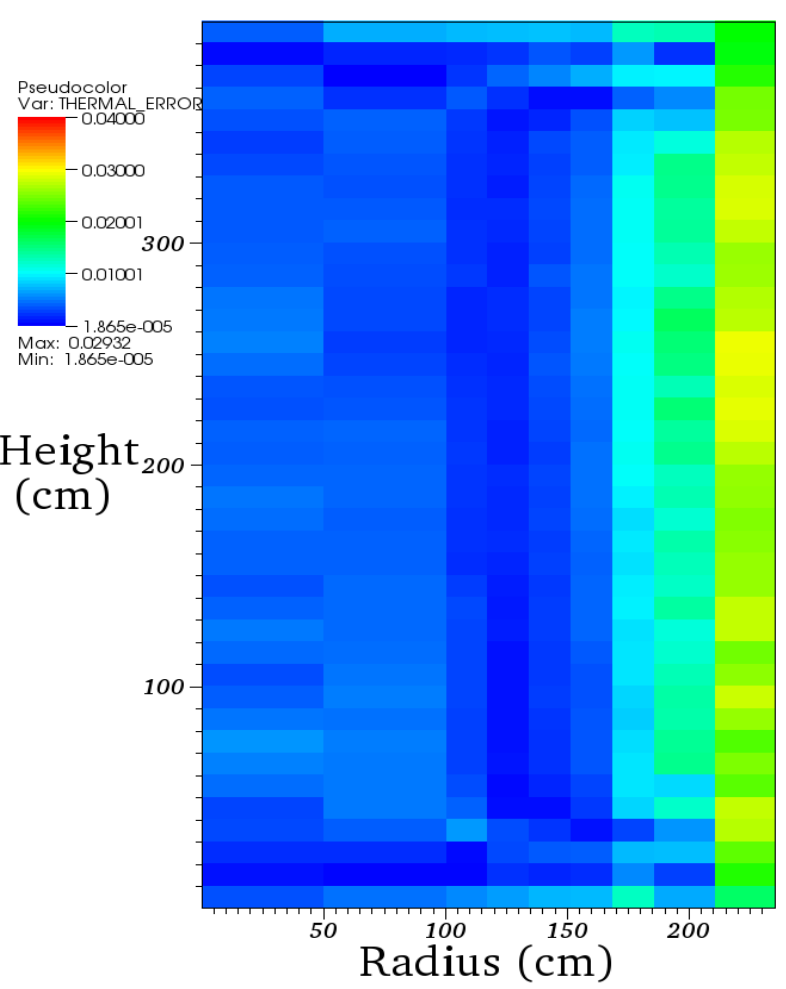

Figure 3. Plot of the relative error of the thermal flux for the hybrid method vs. the MCNP reference solution. 


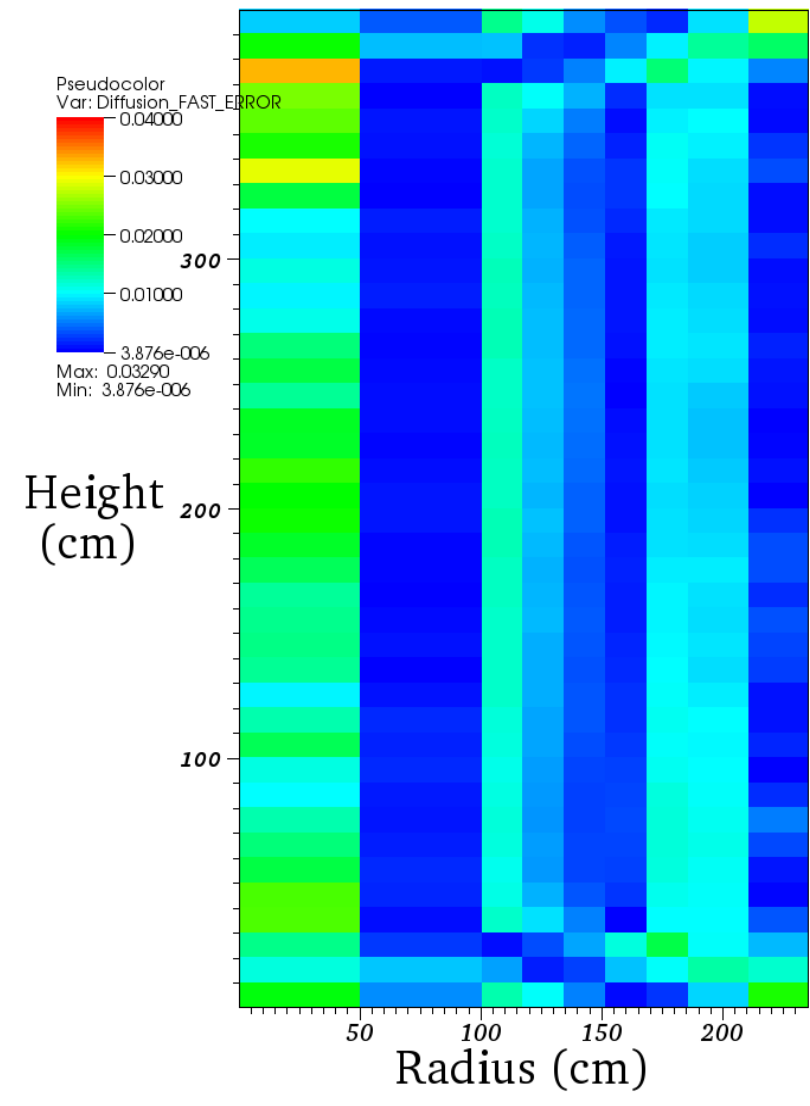

Figure 4. Relative error the fast flux for the pure diffusion method vs. the MCNP reference solution.

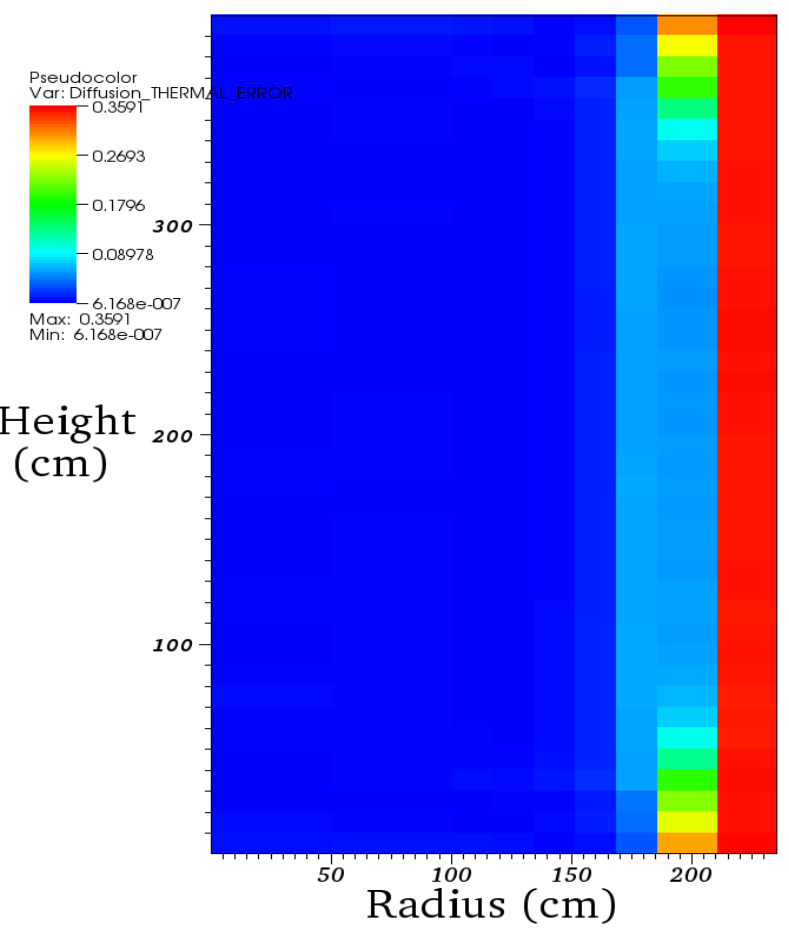

Figure 5. Relative error for the thermal flux for the pure diffusion method vs. the MCNP reference solution.

\subsection{Results for the 2-D R-Theta Geometry}

To demonstrate the capturing of the anisotropic effects of the control rods and boundary in the 2-D R-Theta geometry, a simple mock PBMR core with fully inserted control rods was modeled. The reactor core is a cylinder of infinite height. The reactor core has a total radius of $235 \mathrm{~cm}$ and is made of three basic regions. The first region is an inner reflector with a radius of $100 \mathrm{~cm}$, the second region is fuel ring that is $85 \mathrm{~cm}$ long, and the third region is an outer reflector that is $50 \mathrm{~cm}$ long. A simple diagram of the reactor is shown in Figure 6.

In Figure 6, the inner blue zones are the inner reflector, the green zone is the core fuel, and the red zones are the outer reflector. The deep red zone or first outer reflector region contains 24 control rods. These are equally spaced throughout the outer reflector region close to the core fuel zone. A reference solution was generated by MCNP for comparison.

Moreover, the hybrid method was examined with two different couplings to the incoming current of the designated diffusion region. In all cases the diffusion region was designated to be the inner reflector and the core region and the transport region was designated to be the control rod and outer reflector. The first coupling was obtained from the use of albedos generated from a MCNP calculation, and the second coupling was obtained from an embedded code that uses response functions. The results of the core eigenvalue are shown in Table 2. 


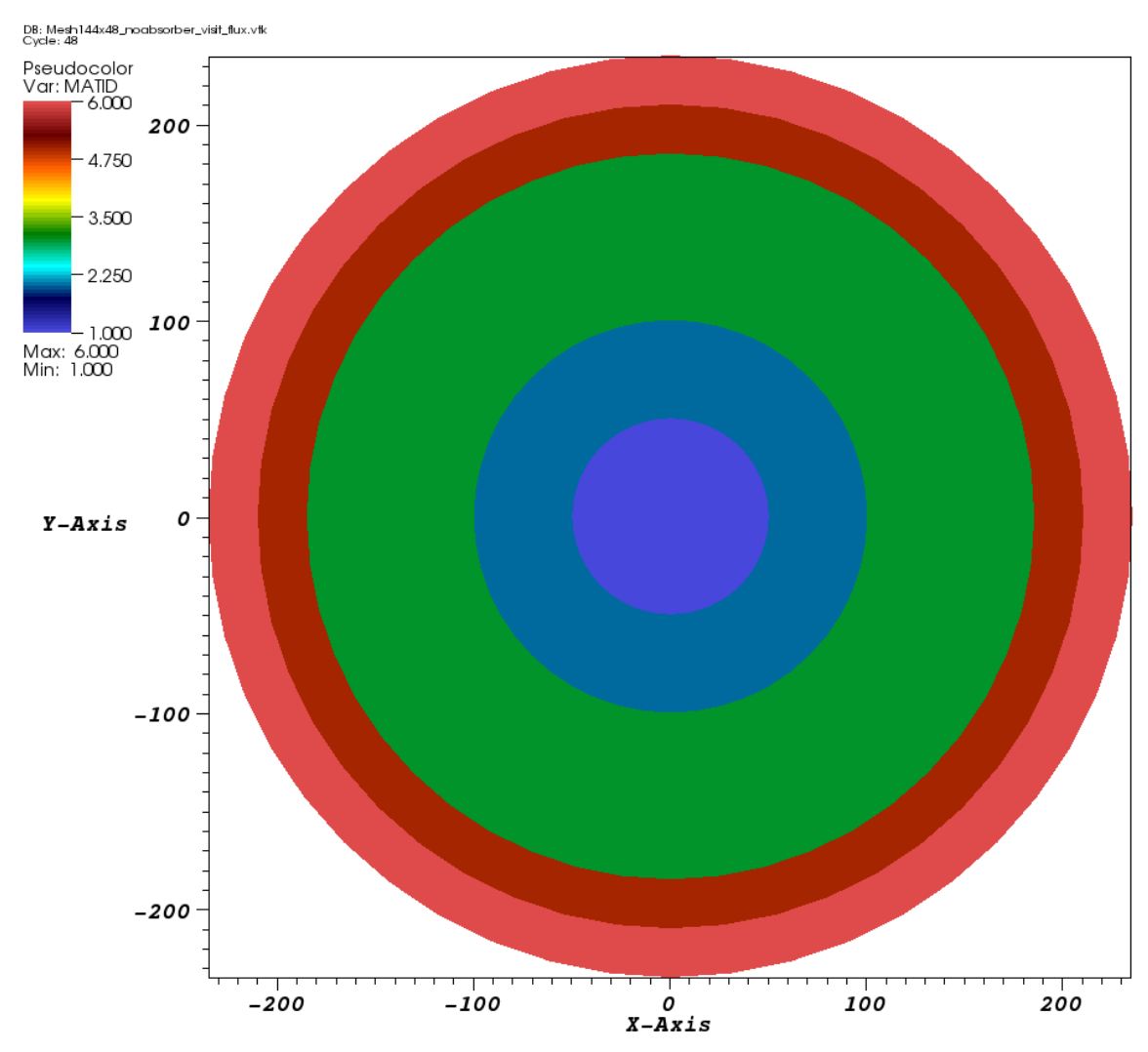

User: GLEIFN

Thu Apr 22 16:23:41 2010

Figure 6. Diagram of the top view of the PBMR core with 24 control rods inserted.

Table 2. Critical core eigenvalues for a fully shutdown case with 24 control rods inserted.

\begin{tabular}{|l|c|}
\hline \multicolumn{1}{|c|}{ Case } & Core Eigenvalue \\
\hline Reference & 0.97515 \\
\hline Pure Diffusion & 0.98192 \\
\hline Albedoes & 0.97498 \\
\hline Hybrid & 0.97443 \\
\hline
\end{tabular}

Table 2 shows that the albedos yields a critical core eigenvalue with a relative error of less than $0.02 \%$, the response function based hybrid method has a relative error less than $0.08 \%$, and the pure diffusion solution gives a critical core eigenvalue that has a relative error that is less than $0.7 \%$. As expected, the small amount of discretization error in the hybrid method gives an eigenvalue that is slightly worse than that of the albedos. Also as expected, the diffusion solution over-predicts the critical core eigenvalue, since it cannot capture the exact angular effects that occur in the control rod region. 

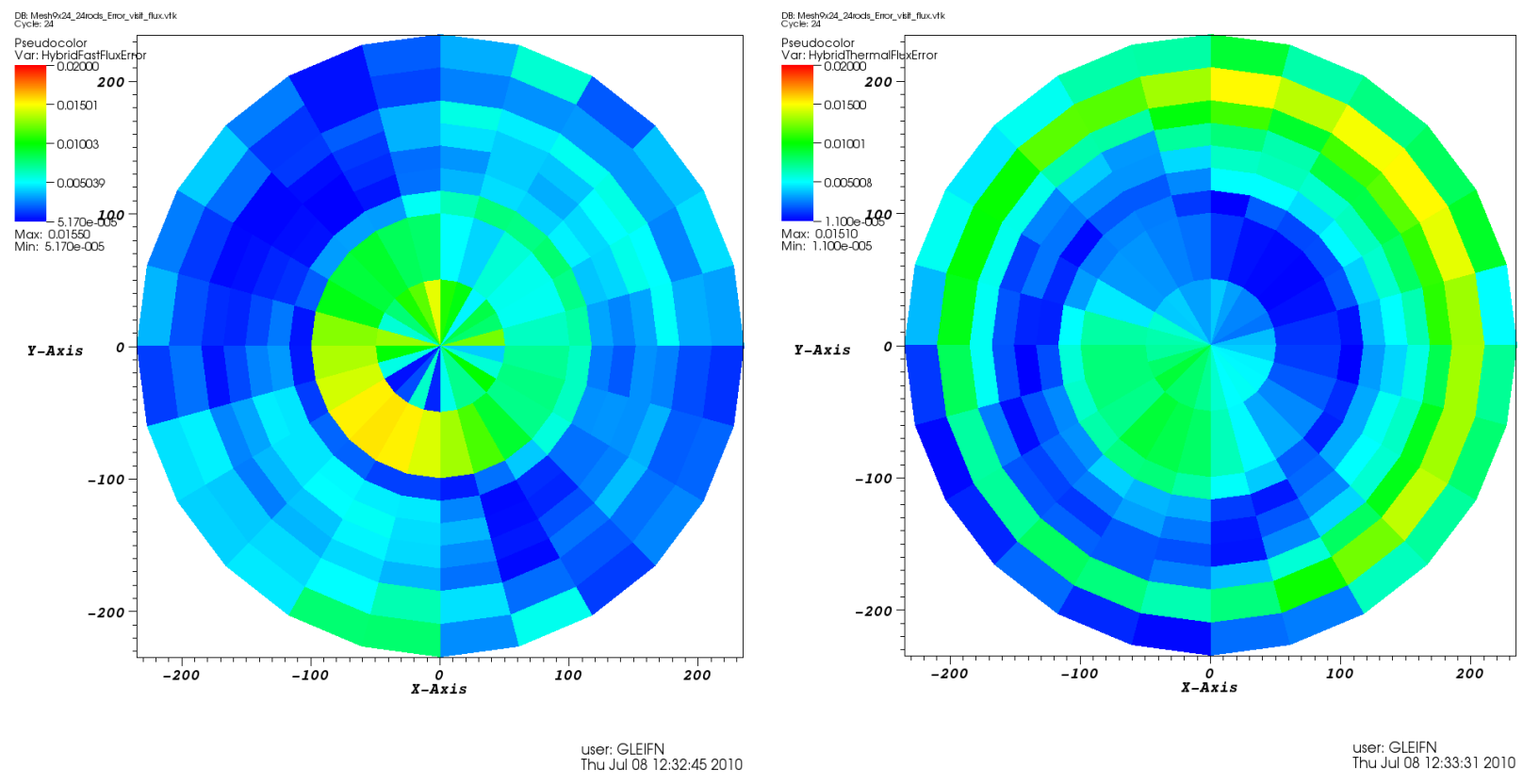

Figure 7. Plots of the relative errors for the fast and thermal energy group scalar fluxes with 24 control rods inserted. The relative errors are obtained by comparing the scalar fluxes generated by MCNP and the fluxes generated from CYNOD Hybrid.
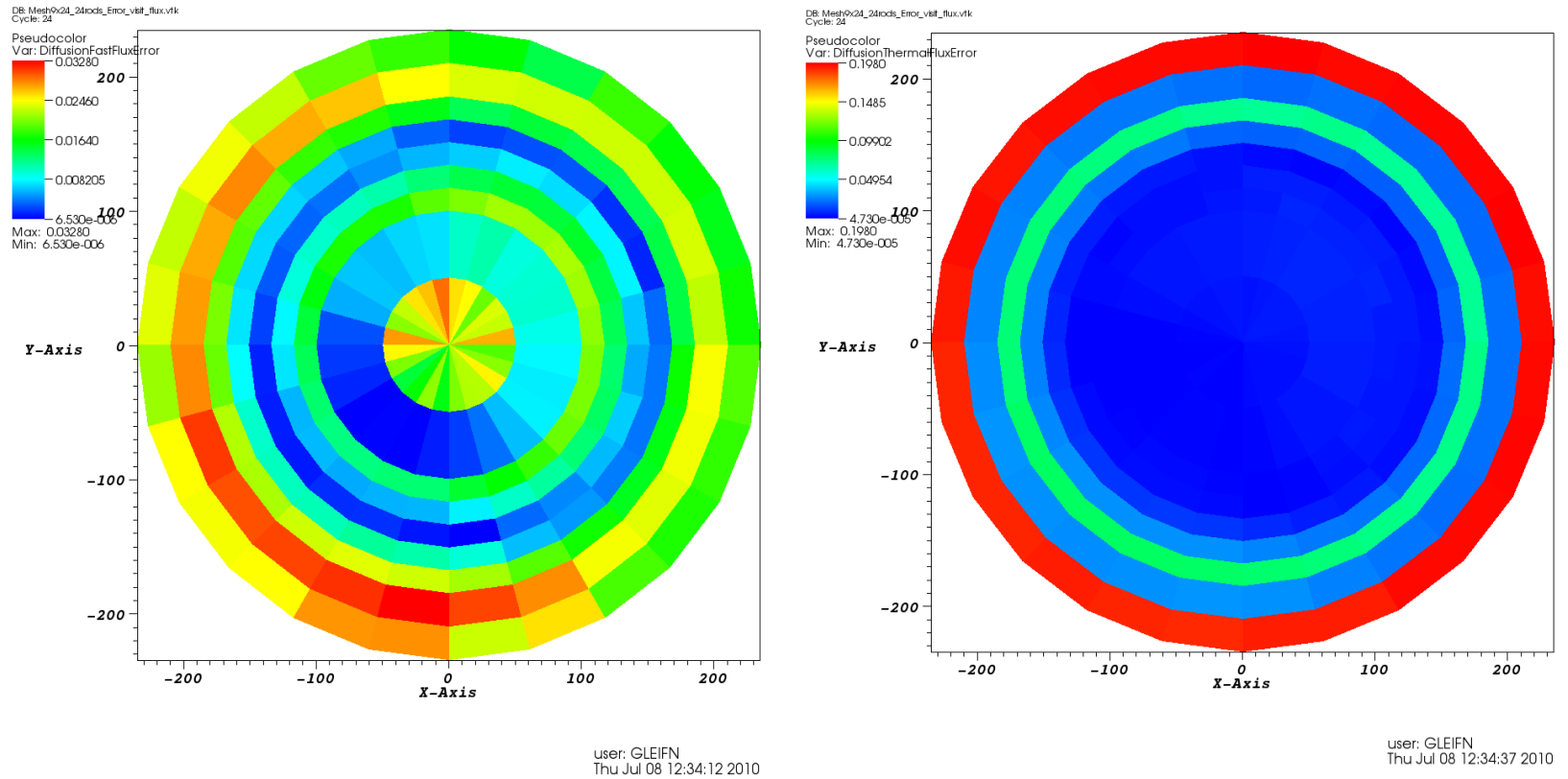

Figure 8. Plots of the relative error of the pure diffusion solution with 24 control rods inserted. The pure diffusion solution obtained from CYNOD is compared to an MCNP solution for the fast and thermal scalar fluxes.

Furthermore, Figures 7 and 8 show the relative errors obtained from a comparison with MCNP. As expected the relative error of the hybrid solution is much less than that of the diffusion solution. The pure diffusion solution for the thermal energy group has a relative error above $19 \%$ in the outer reflector, while the relative error for the hybrid solution is less than $2 \%$ for all regions. The diffusion solution in the fast 
energy group shows a relative error of about $3 \%$ in the control rod region, while the relative error for the hybrid solution is less than $2 \%$ in all regions.

The method was also tested with 12 control rods evenly distributed in the reflector region. A diagram of this core configuration is shown in Figure 9 where the inner blue zones are the inner reflector, the green zone is the core fuel, and the two outer zones are the outer reflector. The red zones in the first outer reflector region are the locations of the 12 equally-spaced control rods. Results for the critical core eigenvalue are given in Table 3.

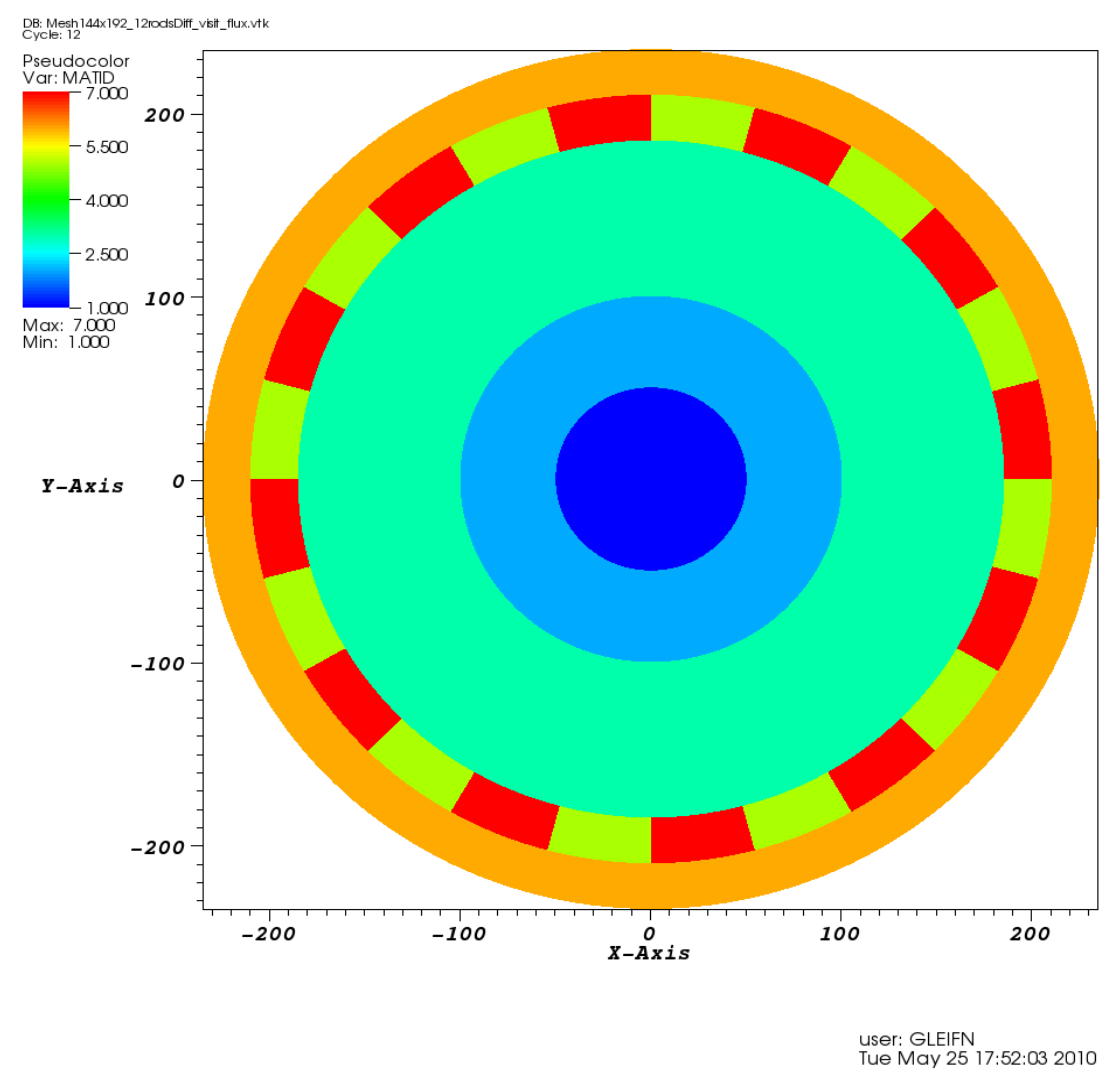

Figure 9. Diagram of the reactor core with twelve control rods inserted in the reflector region.

Table 3. Eigenvalue results for 12 control rods symmetrically inserted into the outer reflector region.

\begin{tabular}{|l|c|}
\hline \multicolumn{1}{|c|}{ Case } & Core Eigenvalue \\
\hline Reference & 1.01597 \\
\hline Pure Diffusion & 1.01400 \\
\hline Albedos & 1.01505 \\
\hline Hybrid & 1.01550 \\
\hline
\end{tabular}

For the 12 evenly-spaced control rods in a critical core eigenvalue, the albedos had a relative error of $0.091 \%$, the hybrid method had a relative error of $0.05 \%$, and the pure diffusion solution had a relative error of $0.194 \%$. The slightly larger error in the critical core eigenvalue for the albedos is because of the statistical nature of the albedo generation. A greater number of histories for the Monte Carlo calculation have to be run to lower the statistical error in the albedos. However, as expected both the albedoes and hybrid method obtains critical core eigenvalues that are superior to pure diffusion. 

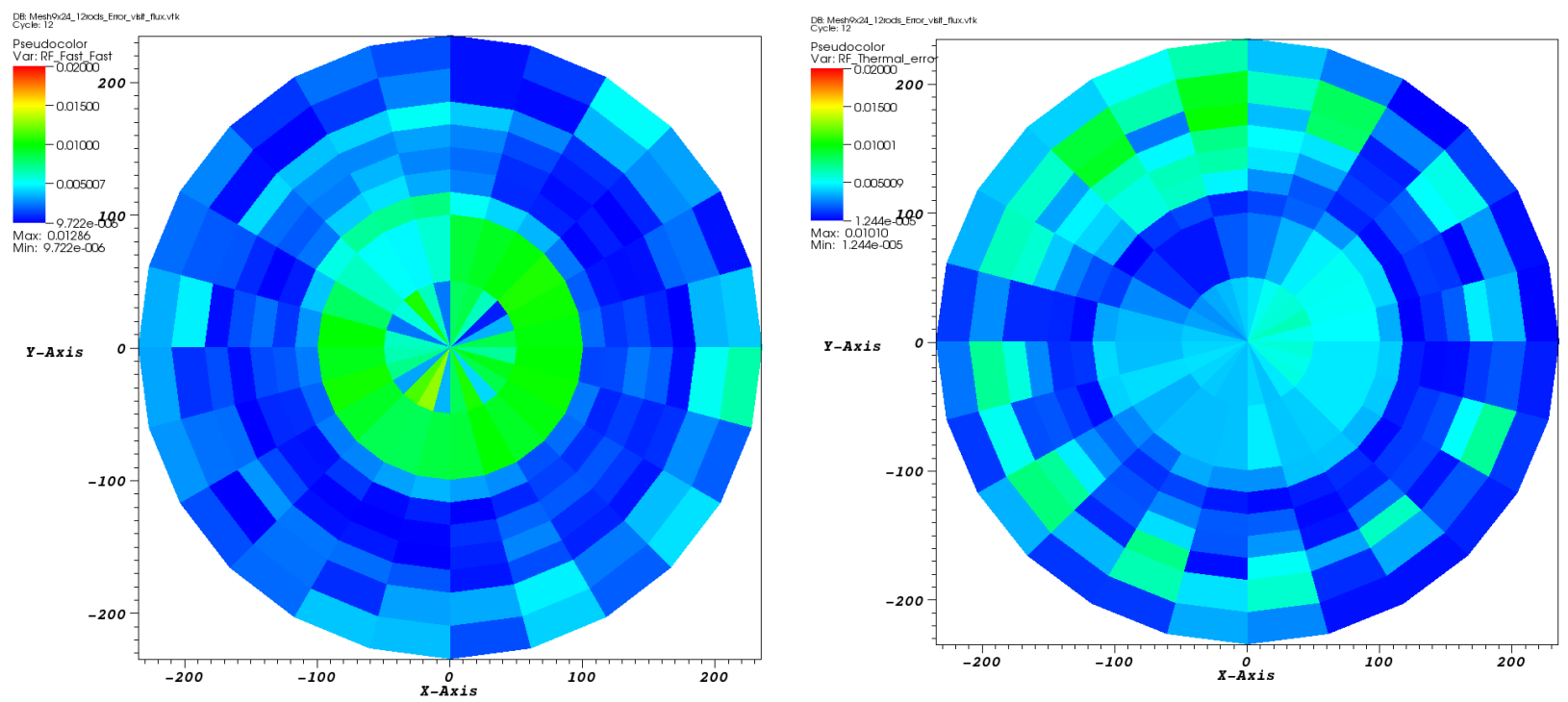

Figure 10. Plots of the relative errors for the fast and thermal energy group scalar fluxes for 12 control rods inserted into the reflector region. The relative errors are obtained by comparing the scalar fluxes generated by MCNP and the fluxes generated from the hybrid CYNOD.
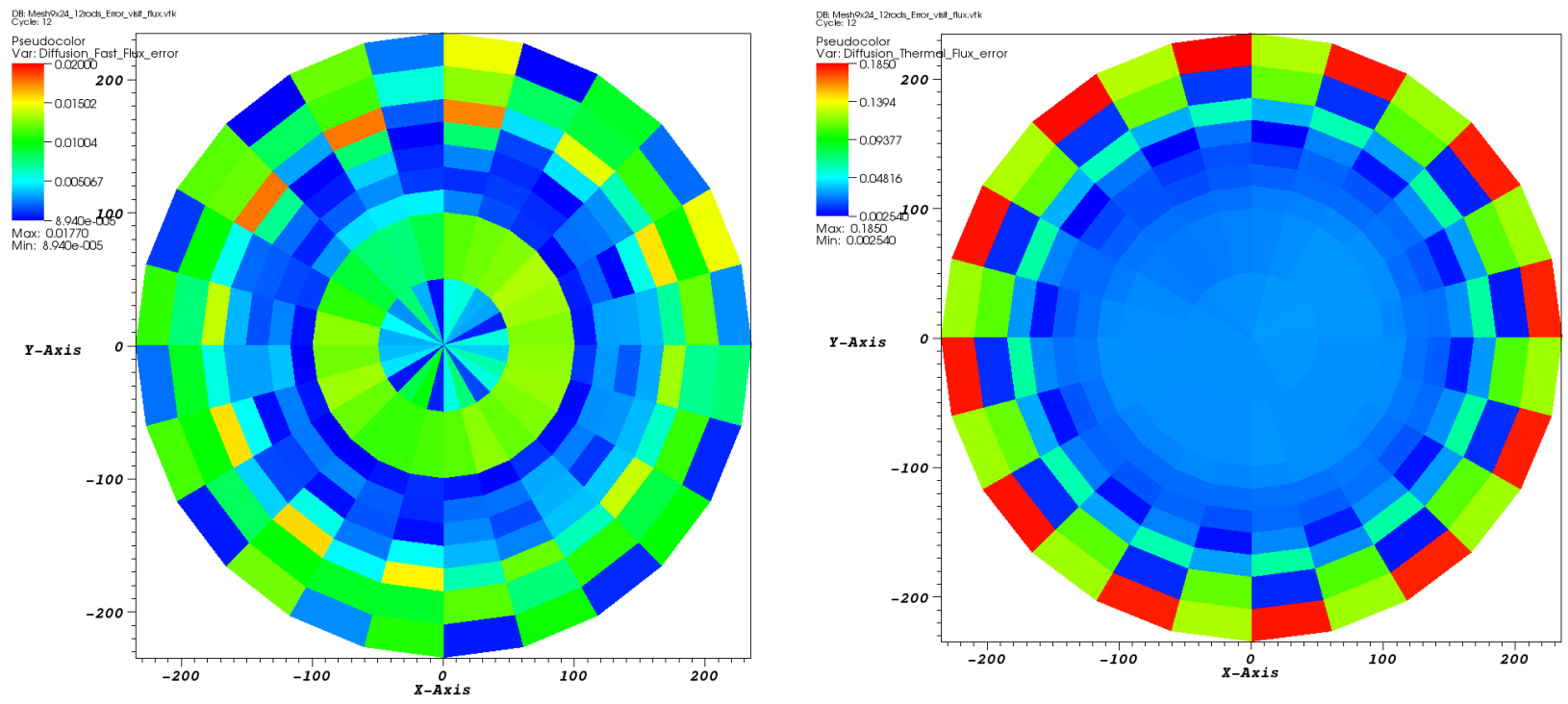

Figure 11. Plots of the relative error of the pure diffusion solution generated by CYNOD compared to a Monte Carlo solution generated by MCNP for the fast and thermal scalar fluxes for twelve control rods inserted into the reflector region.

Figures 10 and 11 also show the relative errors of the computed solution. As expected, the relative error of the hybrid solution is much less than that of the pure diffusion solution. The pure diffusion solution for the thermal energy group has a relative error that is above $18 \%$ while for the hybrid solution has relative errors less than $2 \%$ everywhere. The pure diffusion and hybrid solutions in the fast energy group have relative errors that are less than $2 \%$ everywhere. But the diffusion solution still shows greater error in the control regions when compared to the hybrid solution.

The hybrid methodology was also examined for 12 control rods randomly distributed in the control reflector region. Critical core eigenvalues for the 12 randomly distributed rods are given in Table 4. 
Table 4. Core eigenvalues for 12 control rods randomly distributed.

\begin{tabular}{|l|c|}
\hline \multicolumn{1}{|c|}{ Case } & Core Eigenvalue \\
\hline Reference & 1.02575 \\
\hline Pure Diffusion & 1.02555 \\
\hline Albedoes & 1.02504 \\
\hline Hybrid & 1.02549 \\
\hline
\end{tabular}

Both the diffusion and hybrid methods give critical core eigenvalues that have a relative error of less than $0.03 \%$, while the albedos have a critical core eigenvalue that has a relative error that is less than $0.06 \%$. Again, the greater error in the critical core eigenvalue for the albedos is because of the statistical nature of the albedo generation, and a greater number of histories need to be generated to lower the statistical error. As expected, the hybrid and albedos give good results, but, so do pure diffusion. The diffusion solution does well here because the anisotropic effects become less dominate with the removal of control rod elements.

The fast and thermal flux profiles are shown in Figure 12 for the 12 randomly distributed rods. These plots are generated by the hybrid code. The designated response region gives the average nodal flux for each response node, thus these look like blocks when compared to the refined diffusion region mesh. The rods for this case are distributed randomly but in such a way as to yield a symmetric flux profile across the $y=0$ plane.
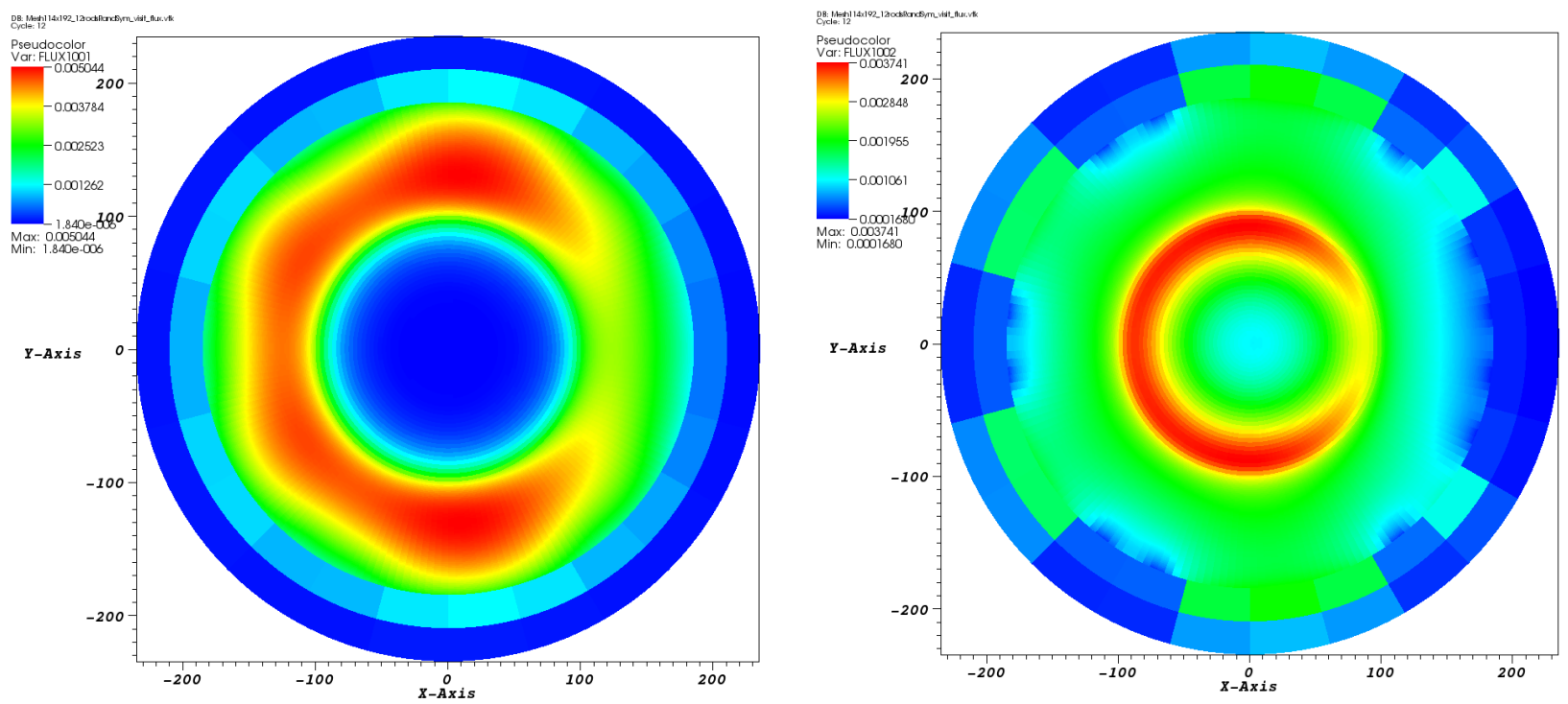

Figure 12. Fast and thermal flux profiles for 12 randomly distributed rods.

The relative error of the hybrid solution, shown in Figures 13 and 14, is smaller than that of the diffusion solution. The pure diffusion solution for the thermal energy group has a relative error of less than $1 \%$ in the core region, which is above $19 \%$ in the outer reflector. The hybrid solution is less than $2 \%$ for all regions. The diffusion solution in the fast energy group shows a relative error of about $3 \%$ in the control rod region while the hybrid solution shows a relative error less than $2 \%$ in all regions. As noted, the relative error in the fast and thermal fluxes for the pure diffusion solution is small in the reactor core region, which means that the critical core eigenvalue for the diffusion solution is very close to the reference value. However, the diffusion solution is not able to adequately capture the anisotropic effects in the outer reflector regions. 

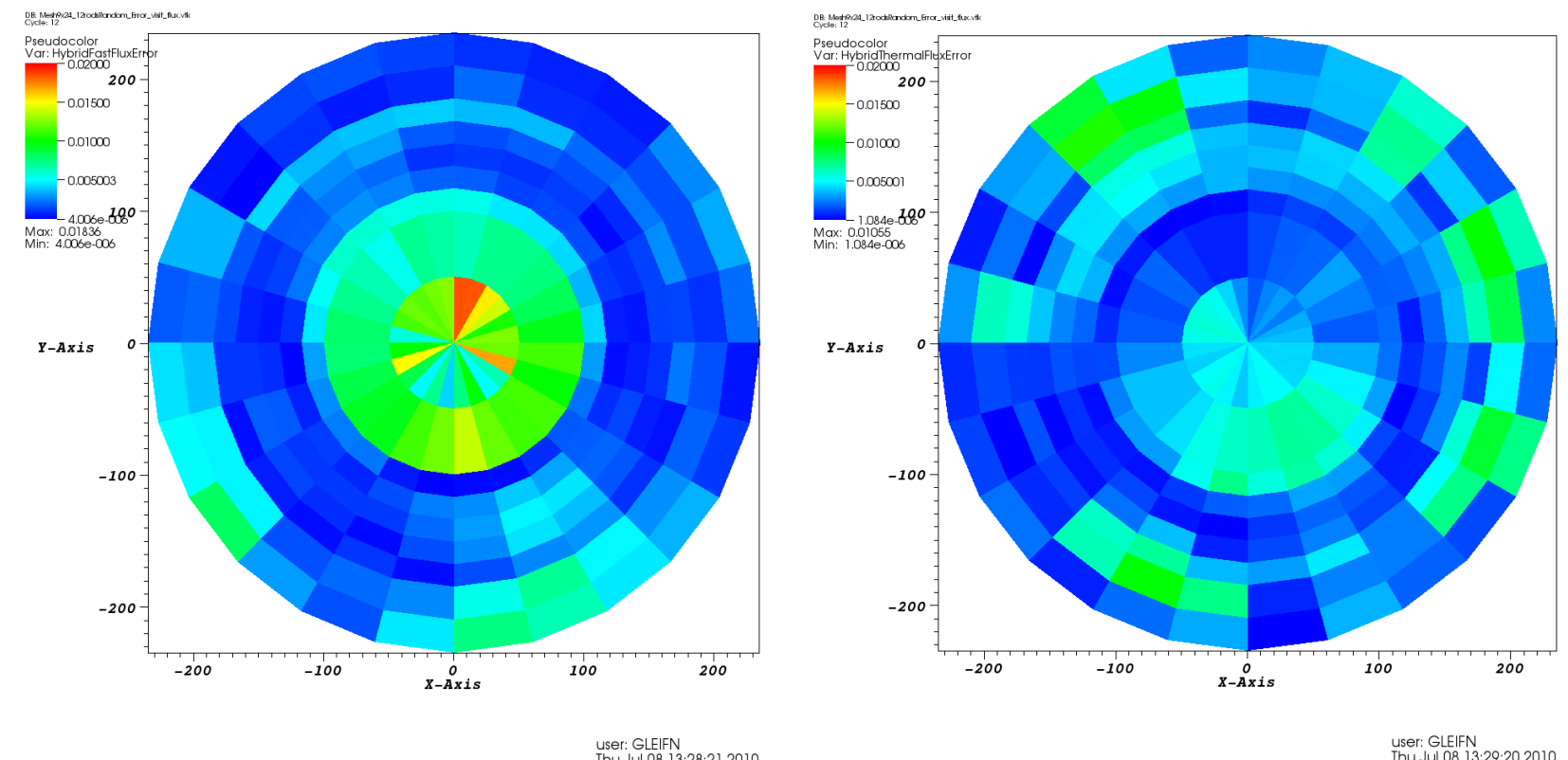

The 140813.28 .212010

User: ELEIFN
Thu Jul 08 13:29:20 2010

Figure 13. Plots of the relative error for the fast and thermal fluxes for 12 randomly inserted control rods. In these plots the Hybrid method is compared to the MCNP.
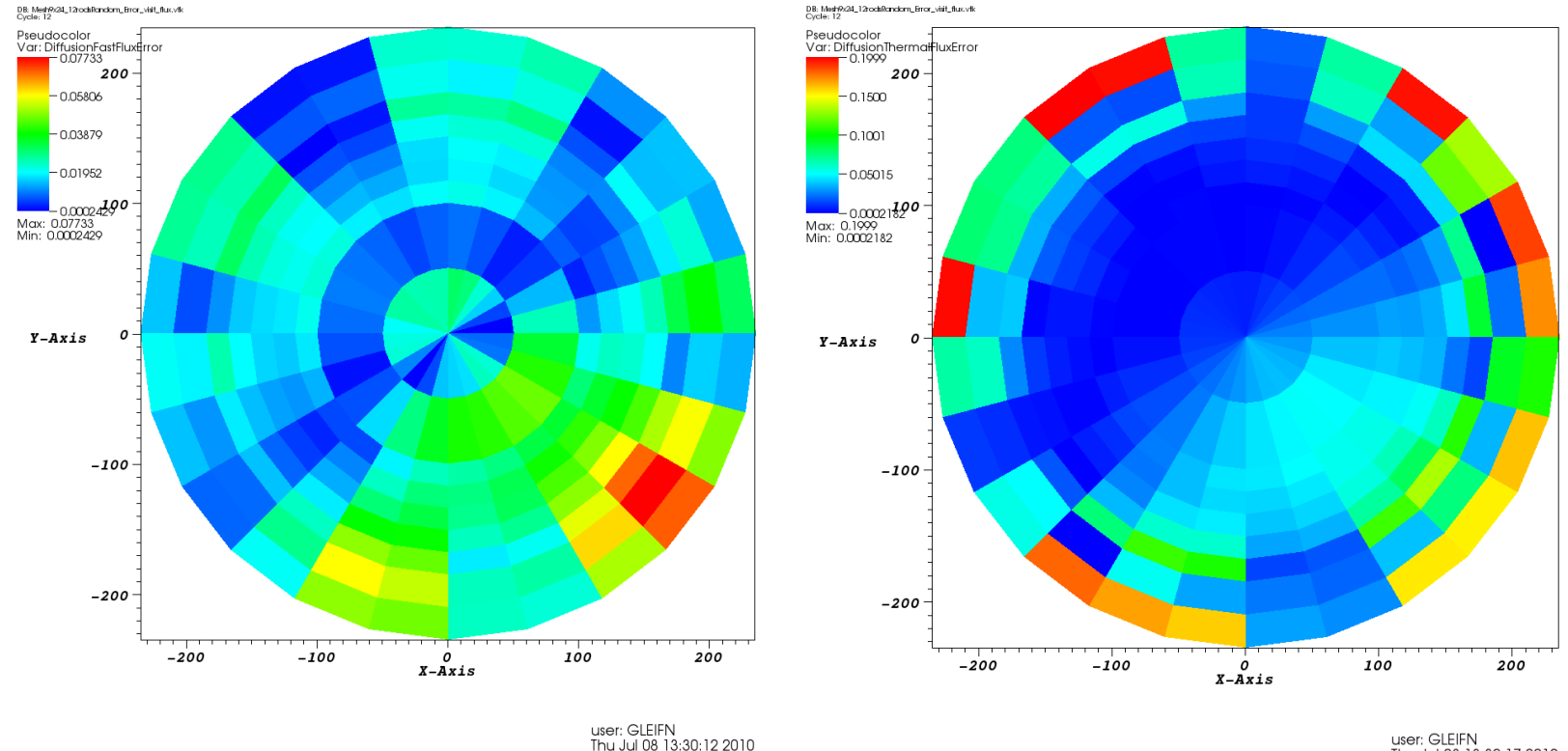

Figure 14. Plots of the relative error of the fast and thermal fluxes with 12 control rods randomly inserted. The relative error was obtained by comparing pure diffusion CYNOD and MCNP.

\subsection{Results for 3-D Geometry}

To demonstrate the capability of the 3-D diffusion method, some initial results and comparisons are presented here. The response function sweeping code and the response functions themselves are currently under development at Georgia Tech, which means that only 3-D results without control rod elements can be accurately modeled at this time. The reactor core is $400 \mathrm{~cm}$ high with a total radius of $235 \mathrm{~cm}$. The fuel region is $350 \mathrm{~cm}$ high and $85 \mathrm{~cm}$ wide, starting by the inner reflector. The inner reflector is $100 \mathrm{~cm}$ wide 
starting at the radial center and the outer reflector is $50 \mathrm{~cm}$ wide. There are no control rod elements present for this sample problem.

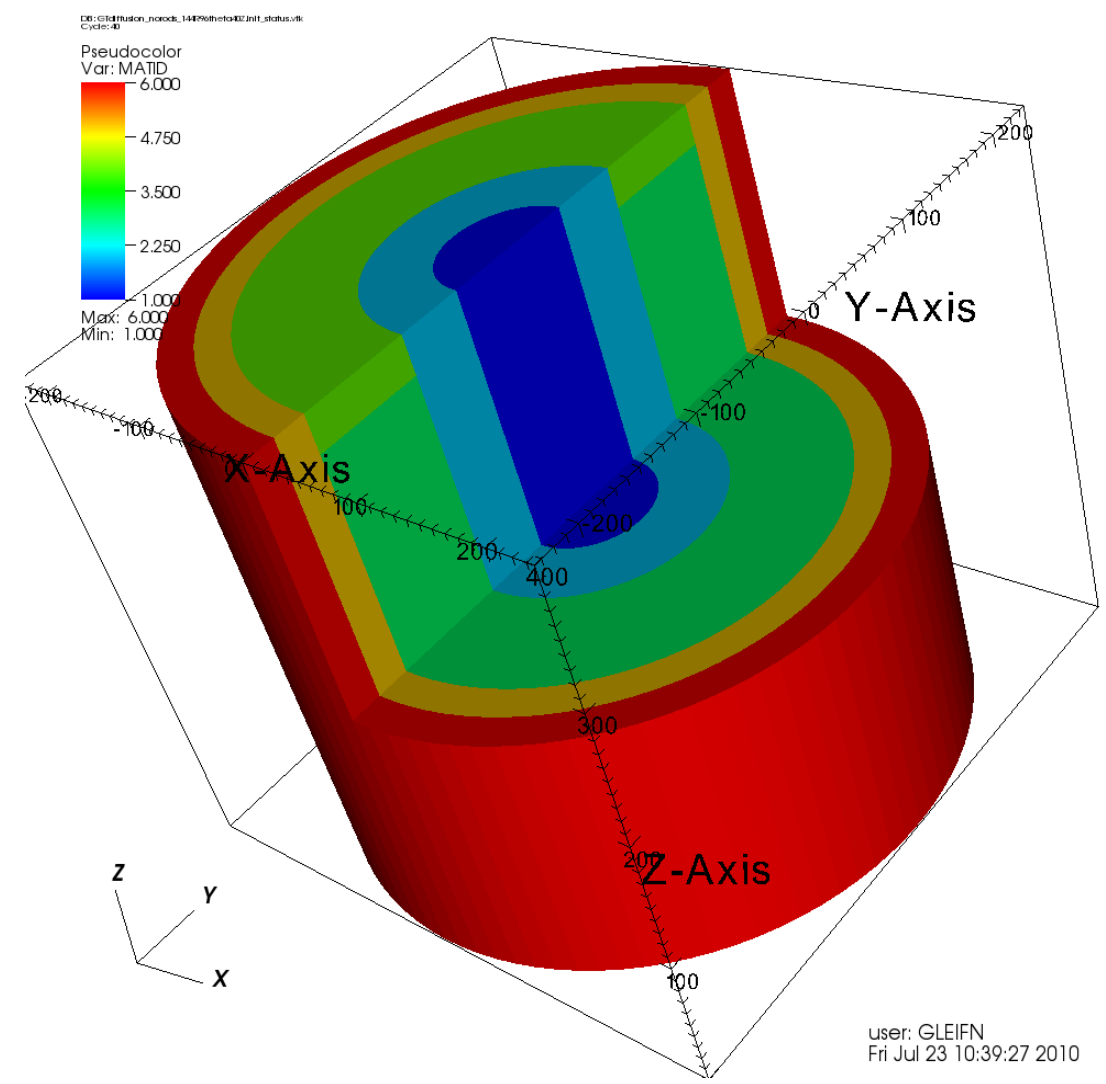

Figure 15. Representation of the 3-D reactor without control rods.

In Figure 15, the inner blue zones are the inner reflector, the green zone is the core fuel, and the red and yellow orange zones are the outer reflector. The result for the critical core eigenvalues are shown in Table 5 .

Table 5. Core eigenvalues for a 3-D problem without control rod elements.

\begin{tabular}{|l|c|}
\hline \multicolumn{1}{|c|}{ Case } & Critical Core Eigenvalue \\
\hline Reference & 1.03720 \\
\hline CYNOD & 1.03732 \\
\hline
\end{tabular}

When the critical core eigenvalues in Table 5 are compared, the error between the reference solution generated by MCNP and 3-D Cynod is less than $0.015 \%$.

The error in the fast and thermal fluxes is plotted in Figure 16, which shows that the error is less than $0.02 \%$ for the core region. Only the fast flux shows an error that is greater than $2 \%$, and this error is located in the very outer edge ring of the reflector boundary. 

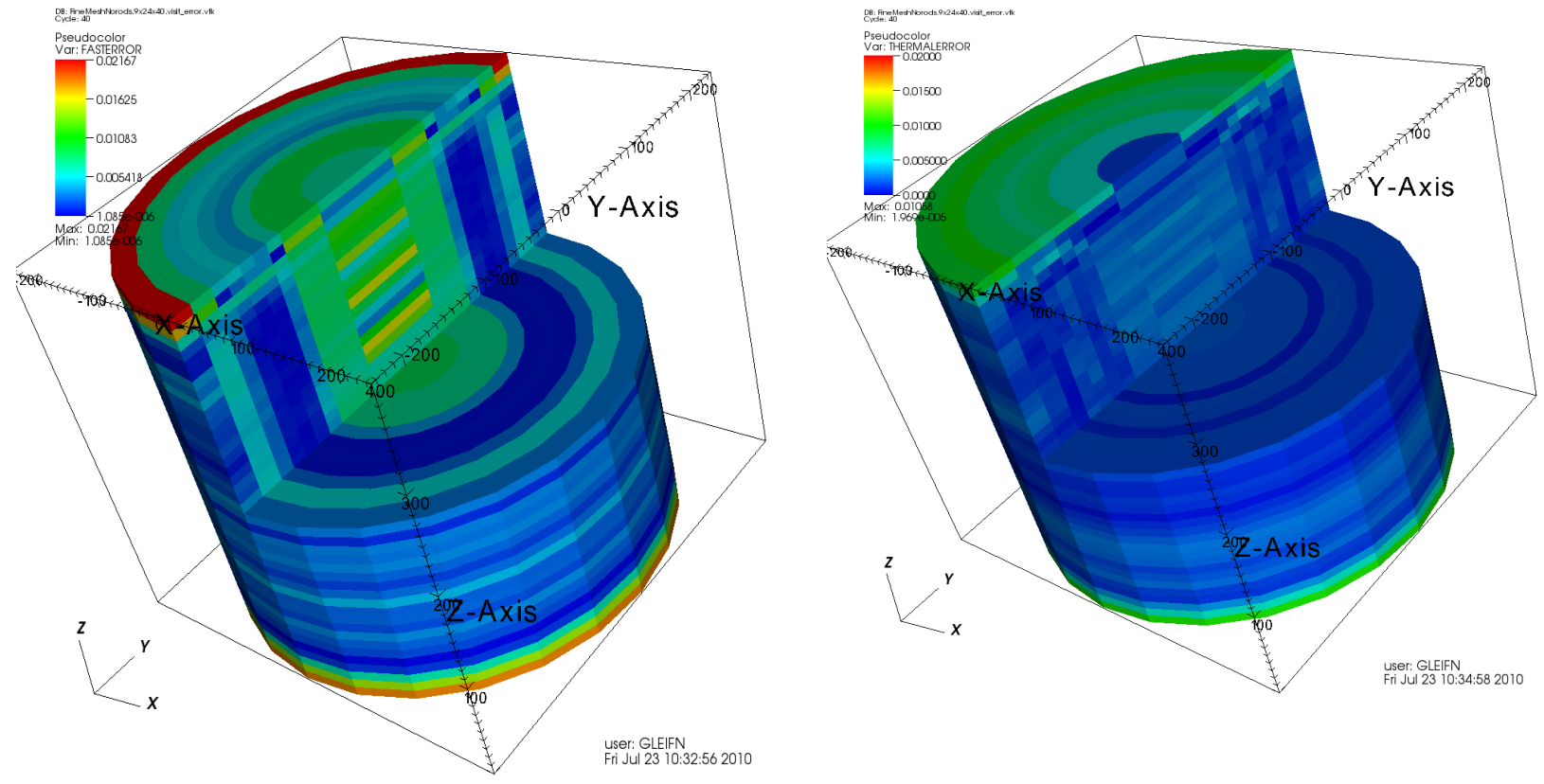

Figure 16. Plots of the error for both the fast and thermal groups. 


\section{TRANSPORT CORRECTION METHOD FOR THE TREATMENT OF BURNABLE POISONS}

Many reactor designs include the presence of BP pins in certain regions of fuel assemblies or of fueled prismatic blocks, which is the case for prismatic-block HTRs. The BP pins are lumps of highly neutron-absorbing material that can cause local flux depressions in their immediate vicinity. Heretofore, such local flux depressions have not been properly captured when modeling the reactor with a diffusionbased nodal method. The failure to properly capture the physics of such localized heterogeneities stems from the very nature of modern nodal methods and the computational paradigm they are based upon. This paradigm is briefly summarized in the following paragraphs.

Modern nodal methods, and their related modern homogenization methods were developed in the late 1970 s and early to mid-1980s. ${ }^{8,14}$ The universally used scheme in modern nodal methods follows the same set of overall steps. First, the material and geometric details at the assembly level are homogenized. The methods for carrying out this homogenization step have substantially evolved and increased in sophistication. However, the most commonly used approach is that of "Modern Equivalence Theory.", 15 Given the homogenized diffusion theory data, the second step is to carry out full core nodal diffusion solution for the flux and effective multiplication factor; part of the core may suffice when symmetries are taken advantage of. This second step is carried out with a variety of codes. Most modern codes are based on the transverse integration procedure that changes the full 3-D diffusion problem into a coupled set of 1-D problems. The solution methods vary from polynomial approaches to analytical ones or even function-expansion-based ones. In all cases, this second step yields the full reactor flux solution. And in all cases the methods follow this two-step approach of full assembly-level homogenization followed by full-core diffusion theory solution. As stated above, this approach homogenizes the BPs as well as the rest of the features within the assembly and effectively distributes the effect (absorption) of the BP uniformly throughout the assembly.

The method presented in this report is a major departure from the conventional approach of modern nodal methods. While the overall two-step scheme is retained, the homogenization step is no longer required to be implemented in its entirety. That is, instead of fully homogenizing all the geometric and material features within an assembly, most of the features are fully homogenized, while the locally confined strong absorbers such as lumped BPs and control elements are retained without homogenization. Instead, compatible diffusion theory data are generated for them. This way a self-consistent set of diffusion theory data is generated for the background homogenized assembly domain and for the explicitly treated strong absorbers.

The novel approach outlined above requires the development of a partial-homogenization method capable of generating background homogenized diffusion theory data for the assembly while simultaneously generating compatible data for the localized strong absorber that is left out of the homogenization process. This novel approach also requires the development of a full-core nodal method that is capable of the explicit treatment of local heterogeneities. Such a method is a new development and has never been reported in the literature. Past nodal methods were developed that treated explicitly mild heterogeneities such as those induced by depletion or by smooth variations in local temperature ${ }^{16}$ and that can be rendered by a low order polynomial representation with reasonable accuracy. Such older methods have even been applied to the high-fidelity modeling of realistic depletion problems. ${ }^{17}$ The new requirement for the explicit modeling within the nodal solver of a localized strong lumped absorber departs significantly from such smooth situations.

The focus of this section is the development of the explicit treatment of heterogeneities in nodal methods and the associated incomplete homogenization method. First, the underlying theoretical developments are introduced, followed by a demonstration of the combined use of the two new methods on a sample problem in which BPs are located near some of the vertices of a prismatic fuel block in a 
model ("toy") HTR representative of the actual HTR being considered for the NGNP. The obtained results show that the new paradigm provides an effective means of treating BPs. 


\section{THEORY OF EXPLICIT TREATMENT OF STRONG HETEROGENEITIES IN NODAL DIFFUSION SOLUTIONS}

This section summarizes the three main theoretical developments used in the incomplete homogenization approach: a proposed mathematical model for the BP, theoretical considerations derived from the rigorous nodal balance equation, and the generation of incomplete homogenized parameters, which reintroduce the spatial heterogeneity within the homogenized node. The technique presented in this work is, in theory, compatible with advanced homogenization techniques, such as those introduced by Generalized Equivalence Theory. ${ }^{8,18}$

\subsection{Burnable Poison Model}

Consider the integrated reaction rate because of absorption occurring inside a finite-dimensional subdomain of volume $V^{p}$, henceforth referred to as a $B P$, surrounded by an infinitely homogenous material containing a time-independent distributed source that emits mono-energetic neutrons as

$R_{a}^{p}=\int_{V^{p}} \Sigma_{a}(\vec{r}) \varphi(\vec{r}) d V$

where $\varphi(\vec{r})$ represents the neutron flux as a function of space, $\Sigma_{a}(\vec{r})$ is the macroscopic absorption cross-section for the whole domain, $\vec{r} \in \mathbf{R}^{3}$, and all other notation, unless otherwise noted, is standard. Under the assumption that the macroscopic absorption cross-section inside the BP is constant, and defining the average flux over the absorbing region is

$\bar{\varphi}^{p} \equiv \frac{1}{V^{p}} \int_{V^{p}} \varphi(\vec{r}) d V$,

from which the reaction rate caused by absorption over the BP in terms of the average flux is obtained as

$R_{a}^{p}=\sum_{a}^{p} V^{p} \bar{\varphi}^{p}$

where $\Sigma_{a}^{p}$ is the constant macroscopic absorption cross-section inside the BP. Any proposed equivalent model for the BP absorption should at least conserve the integrated reaction rate over the subdomain. Considering Equation 16 and assuming space-dependence for the macroscopic absorption rate inside the $\mathrm{BP}$ region, the space-dependent absorption cross-section is defined as

$\Sigma_{a}^{p}(\vec{r})=\delta \Sigma_{a}^{p}\left(\vec{r}-\vec{r}^{\prime}\right)=\left\{\begin{array}{l}+\infty, \vec{r}=\vec{r}^{\prime} \\ 0, \vec{r} \neq \vec{r}^{\prime}\end{array}\right.$

This representation of the absorption cross-section is clearly a mathematical idealization, since the actual macroscopic cross-section is constant over the burnable absorber. This model for the absorption cross-section will be justified by certain advantages that it brings to the nodal approach, as shown later in this report. It is clear that, according to Equation 19, the absorption cross-section behaves as a Dirac delta function (more accurately, a generalized Dirac product), and that the definition presented in the report is merely heuristic. Rigorously, the Dirac delta function is a generalized function which, according to the theory of distributions, is not a function itself but a linear functional on the space of test functions that are smooth over the domain and have compact support. The proposed model for the absorption cross-section for the BP, which collapses the region into a line absorber, can be written as

$\Sigma_{a}^{p}(\vec{r})=\tilde{\Sigma}_{a}^{p} \delta\left(x-x^{\prime}\right) \delta\left(y-y^{\prime}\right)$ 
where $\left(x^{\prime}, y^{\prime}\right)$ indicates the location of the BP, which will be assumed to be constant along the $z$ direction, $\delta\left(X-X^{\prime}\right), X \in(x, y)$ is a symbolic function of the delta distribution, and $\tilde{\Sigma}_{a}^{p}$ is an effective absorption reaction rate that has yet to be determined. In light of Equation 20, the integrated absorption reaction rate is

$$
\int_{V^{B P}} \Sigma_{a}^{p}(\vec{r}) \varphi(\vec{r}) d V=\tilde{\Sigma}_{a}^{p} h^{p} \bar{\varphi}\left(x^{\prime}, y^{\prime}\right)
$$

where $h^{p}$ is the height of the line absorber or collapsed BP, and $\bar{\varphi}\left(x^{\prime}, y^{\prime}\right)$ is the $z$-direction averaged scalar flux evaluated at the location of the BP. In order to conserve the integral reaction rate, we must enforce the following condition on the BP model

$$
\tilde{\Sigma}_{a}^{p} h^{p} \bar{\varphi}\left(x^{\prime}, y^{\prime}\right)=\Sigma_{a}^{p} V^{p} \bar{\varphi}^{p}
$$

Equation 22 implies that $\tilde{\Sigma}_{a}^{p}$ must be determined such that the actual absorption reaction rate (right side of the equation) should be recovered when $\tilde{\Sigma}_{a}^{p}$ is multiplied by the flux at the location of the BP. In order to correctly obtain $\bar{\varphi}^{p}$, an auxiliary transport calculation must be performed and incorporated into Equation 22. In addition, there is no a priori knowledge of $\bar{\varphi}\left(x^{\prime}, y^{\prime}\right)$, since that would require the solution of diffusion problem in order to compute the effective absorption cross-section. In fact, Equation 22 may define a similar method for generating cross-section as superhomogenisation (SPH), ${ }^{18}$ hence nonlinear iterations are required in order to obtain the exact effective cross-section.

As a first approximation, the strategy in this work involves the use of the assembly-averaged flux from the lattice transport calculation to recover the transport reaction rate because of the BP. This choice is justified by the fact that the nodal diffusion calculation ought to produce an assembly-averaged flux that is close enough to the assembly-averaged flux from the heterogeneous transport lattice calculation.

\subsection{The Nodal Balance Equation}

Consider the steady-state multigroup, multidimensional neutron diffusion equation for a homogenous node $V^{k}$ as

$-D_{g}^{k} \vec{\nabla}^{2} \varphi_{g}^{k}(\vec{r})+\sum_{r, g}^{k}(\vec{r}) \varphi_{g}^{k}(\vec{r})=Q_{g}^{k}(\vec{r})$

where

$$
Q_{g}^{k}(\vec{r}) \equiv F \varphi_{g^{\prime}}^{k}(\vec{r})
$$

and

$$
F \equiv\left(\frac{\chi_{g}}{k} \sum_{g^{\prime}=1}^{G} v \Sigma_{f, g^{\prime}}^{k}+\sum_{g^{\prime} \neq g} \sum_{s, g g^{\prime}}^{k}\right)
$$

and where $D_{g}^{k}$ is the direction-independent diffusion coefficient, and $\sum_{r, g}^{k}(\vec{r}), v \Sigma_{f, g^{\prime}}^{k}$, and $\Sigma_{s, g g^{\prime}}^{k}$ are the removal, fission, and scattering macroscopic cross-sections, respectively. In addition, $\chi_{g}$ is the fission spectrum and $k$ is the effective multiplication factor. Note that the removal cross-section is spacedependent within the node and reduces to the absorption cross-section for the particular case of $G=1$. The diffusion equation is subject to general albedo boundary conditions at the edge of the domain. 
Beginning by operating on Equation 23 with $\frac{1}{V^{k}} \int_{V^{k}} d V$, defining the following quantities

$\bar{\varphi}_{g}^{k} \equiv \frac{1}{V^{k}} \int_{V^{k}} \varphi_{g}^{k}(\vec{r}) d V$

and

$\bar{Q}_{g}^{k} \equiv \frac{1}{V^{k}} \int_{V^{k}} Q_{g}^{k}(\vec{r}) d V$,

and applying Green's theorem to the leakage term, yields

$$
\begin{aligned}
& \frac{1}{V^{k}} \int_{V^{k}} d V\left(-D_{g}^{k} \vec{\nabla}^{2} \varphi_{g}^{k}(\vec{r})\right)= \\
& \sum_{i=1}^{I} \frac{1}{V^{k}} \int_{A_{i}^{k}} d A_{i}\left(-D_{g}^{k} \hat{n}_{i} \cdot \vec{\nabla} \varphi_{g}^{k}(\vec{r})\right)
\end{aligned}
$$

where $\hat{n}_{i}$ is one of the normal vectors in each of the $i$ directions of the surface leakage. The surfaceaveraged leakages in Equation 13-28 can then be defined as

$$
L_{g i}^{k} \equiv J_{g i}^{k}(h)-J_{g i}^{k}(-h)
$$

Note that the removal term on the left hand side of Equation 23 is space-dependent and involves $(P+1)$ terms, where $P$ denotes the total number of BP rods, inside node $k$ and $b$ denotes the removal because of the homogenized background material, i.e. $\sum_{r, g}^{k}(\vec{r})=\sum_{r, g}^{k, b}(\vec{r})+\sum_{p=1}^{P} \sum_{r, g}^{k, p}(\vec{r})$. If operating over this removal term in the diffusion equation with $\frac{1}{V^{k}} \int_{V^{k}} d V$, we obtain

$$
\begin{aligned}
& \frac{1}{V^{k}} \int_{V^{k}} \sum_{r, g}^{k}(\vec{r}) \varphi_{g}^{k}(\vec{r}) d V= \\
& \left(\frac{V^{b}}{V^{k}}\right) \sum_{r, g}^{k, b} \bar{\varphi}_{g}^{k, b}+\sum_{p=1}^{P}\left(\frac{V^{p}}{V^{k}}\right) \sum_{r, g}^{k, p} \bar{\varphi}_{g}^{k, p}
\end{aligned}
$$

where $\bar{\varphi}_{g}^{k, b}$ and $\bar{\varphi}_{g}^{k, p}$ denote the spatially-averaged flux over the background and poison regions, respectively. Note that no approximation has been introduced so far in the derivation. In fact, it is not even necessary to separate between the background and poison regions if we define a homogenized removal cross-section such as

$$
\begin{gathered}
\sum_{r, g}^{k} \equiv \frac{1}{V^{k}} \int_{V^{k}} \Sigma_{r, g}^{k}(\vec{r}) \varphi_{g}^{k}(\vec{r}) d V / \bar{\varphi}_{g}^{k}= \\
\left(\frac{V^{b} \bar{\varphi}_{g}^{k, b}}{V^{k} \bar{\varphi}_{g}^{k}}\right) \Sigma_{r, g}^{k, b}+\sum_{p=1}^{P}\left(\frac{V^{p} \bar{\varphi}_{g}^{k, p}}{V^{k} \bar{\varphi}_{g}^{k}}\right) \sum_{r, g}^{k, p}
\end{gathered}
$$


Equation 31 corresponds to the standard flux-weighted homogenization technique; hence there is no difference between the traditional homogenization and the BP treatment with respect to the balance equation. This is expected, since the balance equation must be respected regardless of the particular heterogeneity introduced into the node, as long as the homogenized cross-sections are defined consistently.

Finally, by using the definitions expressed by Equations 28-31, we arrive at the neutron balance equation

$$
\frac{1}{V^{k}} \sum_{i=1}^{I} A_{i} L_{g i}^{k}+\sum_{r, g}^{k} \bar{\varphi}_{g}^{k}=\bar{Q}_{g}^{k}
$$

which requires the evaluation of the net currents in order to evaluate the net leakage terms. Equation 32 is more general than Equation 23 because there is no particular reference to the diffusion approximation (even though the diffusion equation was in fact the starting point). Hence, Equation 32 is a rigorous and general neutron balance over the fuel assembly (or block), which shows that by carefully defining homogenized parameters (such as those in Equation 31) it is possible to exactly account for the intranodal heterogeneities. Unfortunately, it is not possible in practice to define these exact homogenized parameters because they require the a priori knowledge of the flux solution.

The main theoretical development in this section is the relation between the flux-weighted homogenized removal cross-section and the incomplete background and BP homogenized removal crosssections. This relation will respect the neutron balance and provide a basis for the definitions involve in the incomplete homogenization procedure.

\subsection{A Novel Incomplete Homogenization Method for Explicit Treatment of Strong Heterogeneities}

A particular model for the space-dependent removal cross-section was introduced in Section 6.1 and relevant definitions were established in Section 6.2. This section discusses the generation of incomplete homogenized data in the context of available lattice physics methods and data. 
Recall that the neutron balance equation over a homogenized node remains identically the same as in previous nodal formulations. Hence, all homogenized cross-sections must be generated via the following relation (where $x$ represents some particular reaction rate)

$$
\Sigma_{x, g}^{k} \equiv \frac{\int_{V^{k}} \sum_{x, g}^{k}(\vec{r}) \varphi_{g}^{k}(\vec{r}) d V}{\int_{V^{k}} \varphi_{g}^{k}(\vec{r}) d V}
$$

However, note that the numerator in Equation 33 may be divided into multiple regions, because of the linearity of the integral operator, while remaining consistent with the $\Sigma_{x, g}^{k}$ definition, hence Equation 31 .

Thus, if two sets of material and group dependent removal cross-sections are generated for BPs and background material, and these are weigthed according to Equations 16-31, they can be linearly combined to reconstruct a single homogenized cross-section to be used in the balance equation. Additionally, the same homogenized removal cross-section may be split into two components. The first approximation introduced in this methodology assumes that the local absorber removal can be modeled in accordance with Equation 20, hence

$$
\Sigma_{r, g}^{k}(\vec{r})=\tilde{\Sigma}_{r, g}^{k, b}+\sum_{p=1}^{P} \tilde{\Sigma}_{r, g}^{k, p} \delta\left(x-x^{\prime}\right) \delta\left(y-y^{\prime}\right)
$$

where $\tilde{\Sigma}_{r, g}^{k, b}$ and $\tilde{\Sigma}_{r, g}^{k, p}$ remain undefined. To determine these quantities requires that Equation 34 conserves the heterogeneous reaction rates defined by Equation 30, thereby obtaining

$$
\tilde{\Sigma}_{r, g}^{k, b}=\left(\frac{V^{b} \bar{\varphi}_{g}^{k, b}}{V^{k} \bar{\varphi}_{g}^{k}}\right) \Sigma_{r, g}^{k, b}
$$

and

$$
\tilde{\Sigma}_{r, g}^{k, p}=\left(\frac{V^{p} \bar{\varphi}_{g}^{k, p}}{V^{k} \bar{\varphi}_{g}^{k}}\right) \Sigma_{r, g}^{k, p}
$$

Clearly, Equations $35 \mathrm{a}$ and $35 \mathrm{~b}$ define a homogenization approach in which the region-averaged flux from a lattice calculation can be used in conjunction with the assembly-averaged flux to define incompletely homogenized parameters that conserve the heterogeneous reaction rates and satisfy the nodal balance. The final issue that remains to be solved is the incorporation of the proposed spacedependent removal cross-section into a nodal solver.

The space-dependent cross-section model may be substituted into Equation 23 and the resulting expression manipulated into

$$
-D_{g}^{k} \vec{\nabla}^{2} \varphi_{g}^{k}(\vec{r})+\tilde{\Sigma}_{r, g}^{k, b} \varphi_{g}^{k}(\vec{r})=\tilde{Q}_{g}^{k}(\vec{r})
$$

where

$$
\begin{aligned}
& \tilde{Q}_{g}^{k}(\vec{r})=Q_{g}^{k}(\vec{r})- \\
& \sum_{p=1}^{P} \tilde{\Sigma}_{r, g}^{k, p} \delta\left(x-x^{\prime}\right) \delta\left(y-y^{\prime}\right) \varphi_{g}^{k}(\vec{r})
\end{aligned}
$$


Hence, Equation 36 may be solved using standard nodal techniques, such as the Nodal Green's Function Method. ${ }^{19,20,21}$ The transverse-integration procedure allows for the reduction of Equation 36 into a section of directional ordinary differential equations (ODEs) that can readily be solved by the formal inversion of the elliptic operator via the Green's Function Method. The Green's Function Method also allows for the straightforward treatment of the delta functions defined in Equation 37. 


\section{EXPLICIT MODELING OF BPS: NUMERICAL RESULTS}

In order to test the methodology presented above, a simplified case was modeled using the HEXPEDITE code. The model was used to test the theory and verify that the expected neutron physics were preserved. A 2-D mini-reactor core was modeled consisting of seven hexagonal assemblies with $36 \mathrm{~cm}$ flat-to-flat dimensions. In addition, a set of mono-energetic cross-sections were devised in order to obtain a slightly super-critical system. Vacuum boundary conditions were imposed by introducing a zero incoming partial currents and a single BP introduced at the center fuel block. Note that the removal crosssection of this BP was made rather large in order to obtain the desired effect.

First, an eigenvalue calculation was performed in order to obtain an uncontrolled case, as shown in Figure 17. Because of the vacuum boundary conditions, the leakage effect causes the power distribution to be lower at the surrounding assemblies with respect to the center assembly. Once the BP is inserted into the center fuel block, the power distribution becomes lower relative to the surrounding fuel assemblies (see Figure 18). Next, the BP position is changed from the center to the North and subsequently to the South (Figure 19 and Figure 20). It is evident from the results that the peak relative power distribution is opposite (and symmetric) to the location of the BP. This is the effect that is expected from the neutronic behavior of the BP when inserted near the vertices of the hexagonal block. Additional results are shown for six positions (Figure 21 through Figure 26), which further confirm the expected behavior of the $\mathrm{BP}$ and the correct treatment via the methodology presented in this report.

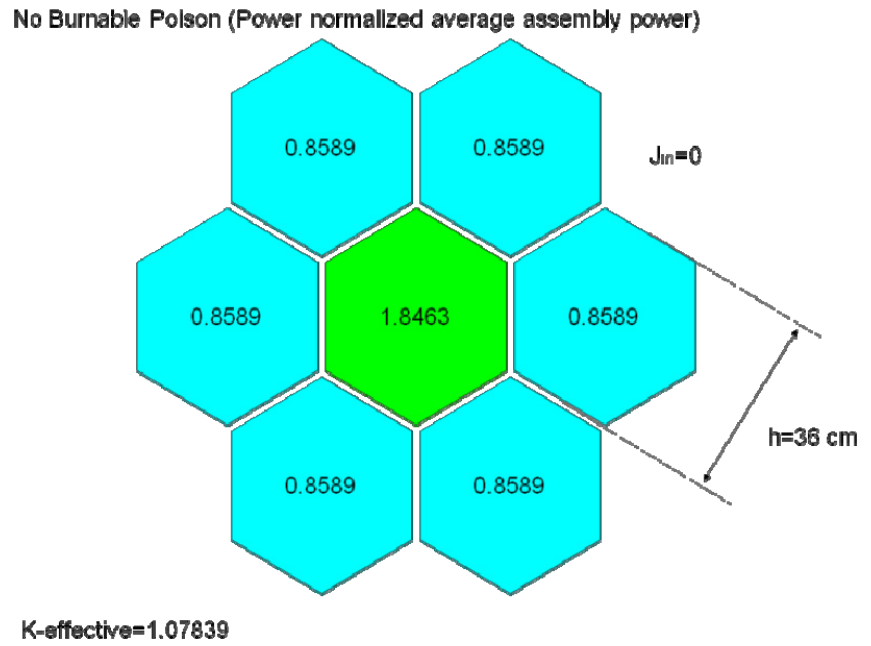

Figure 17. Simplified mini-core effective multiplication factor and power distribution without BP. 


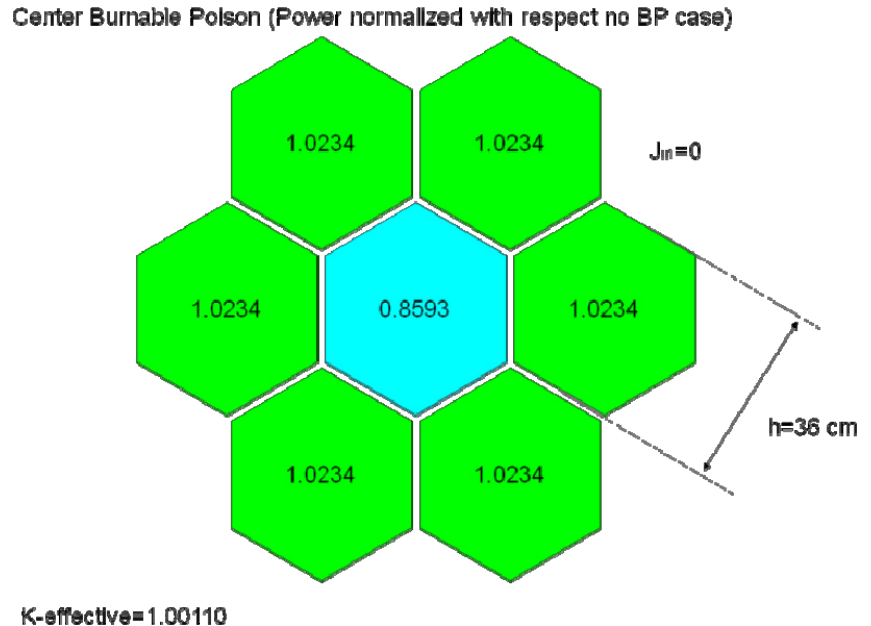

Figure 18. Simplified mini-core effective multiplication factor and power distribution with center BP.

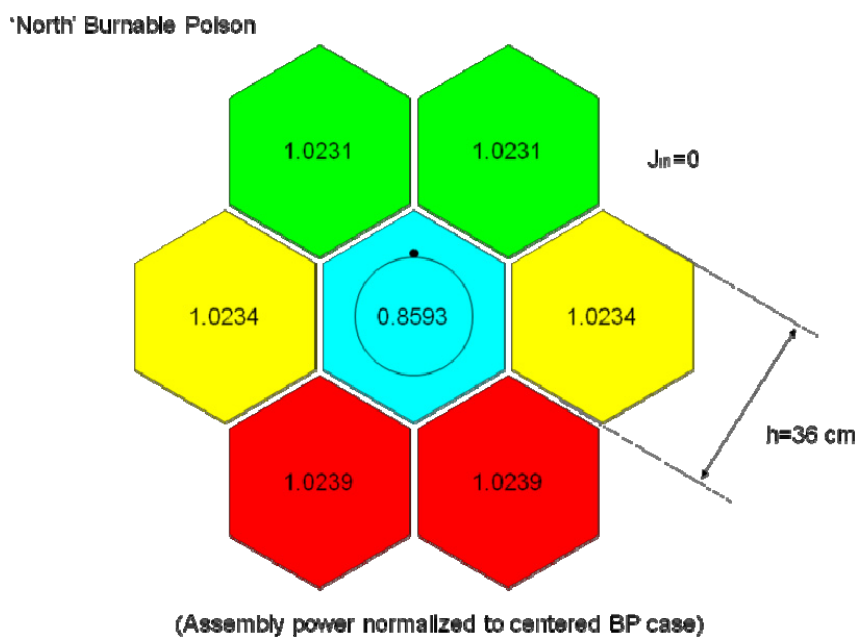

Figure 19. Simplified mini-core effective multiplication factor and power distribution with North BP.

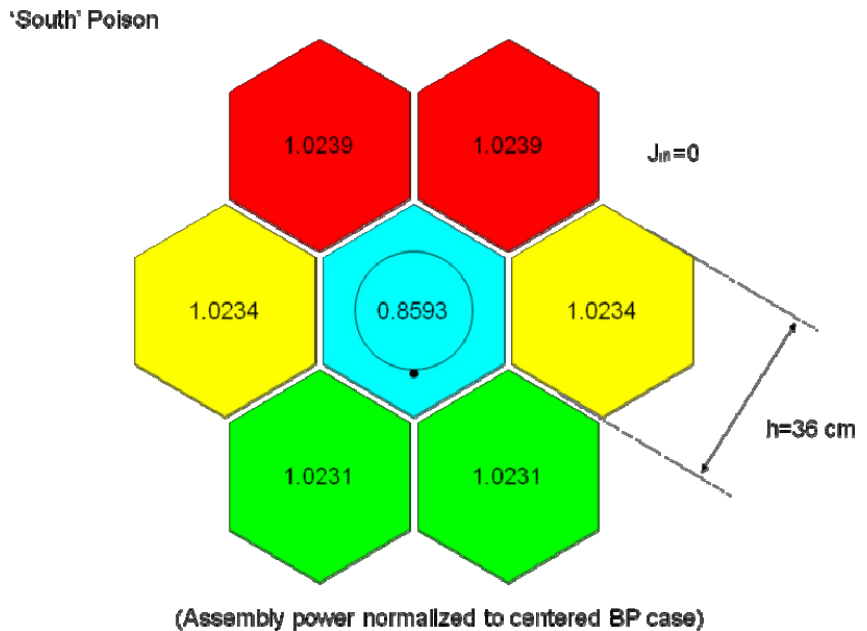

Figure 20. Simplified mini-core effective multiplication factor and power distribution with South BP. 


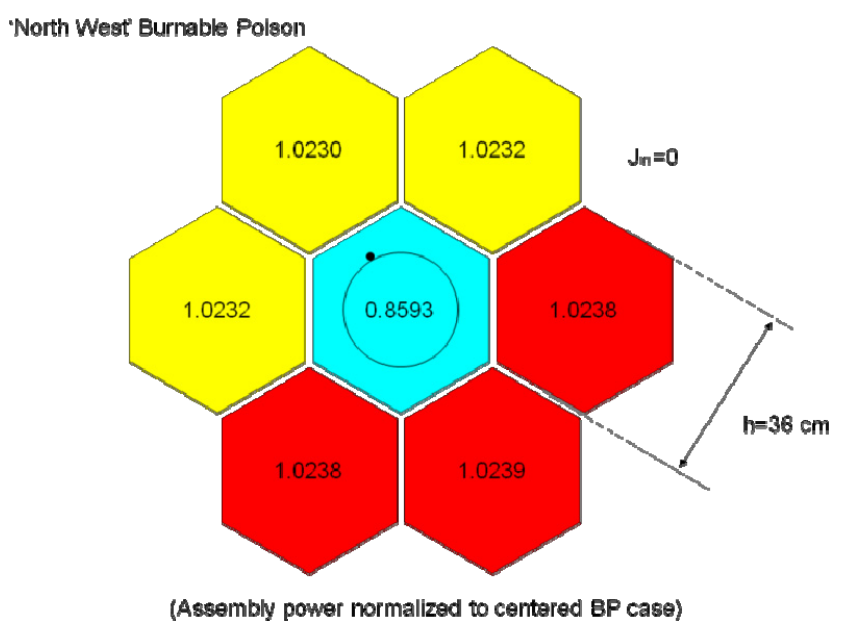

Figure 21. Simplified mini-core effective multiplication factor and power distribution with northwest BP.

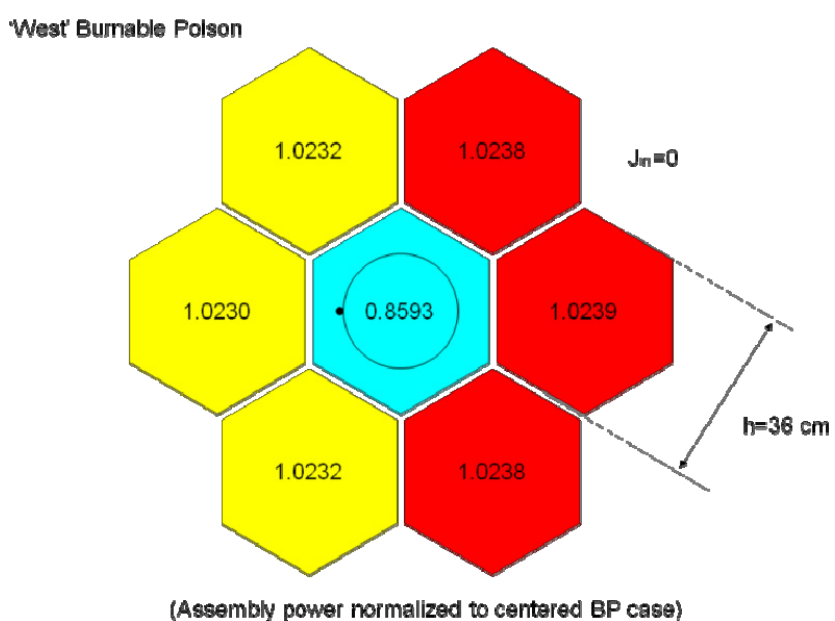

Figure 22. Simplified mini-core effective multiplication factor and power distribution with West BP.

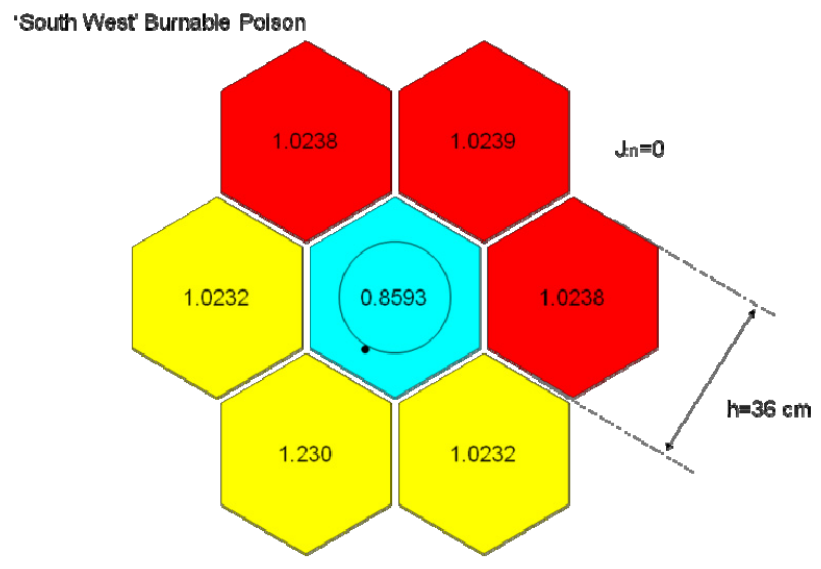

(Assembly power nermallzed to centered BP case)

Figure 23. Simplified mini-core effective multiplication factor and power distribution with southwest BP. 


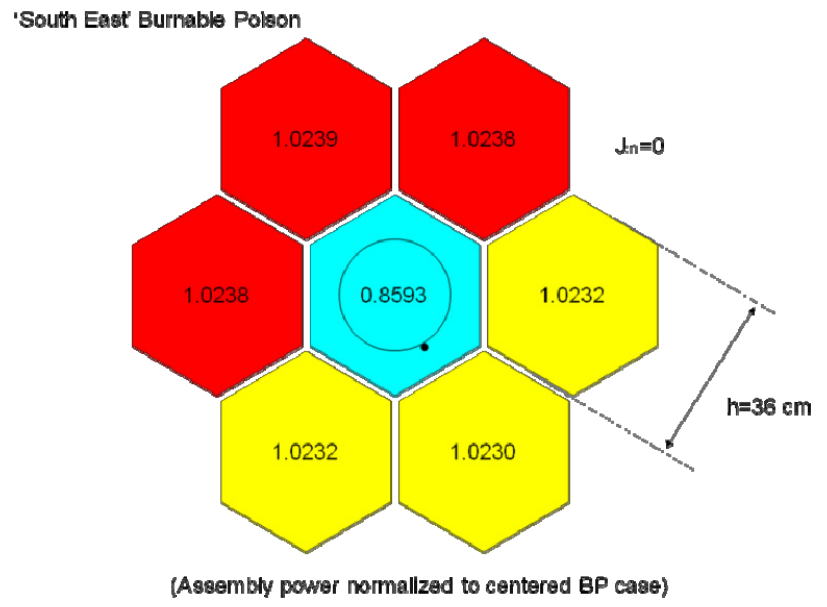

Figure 24. Simplified mini-core effective multiplication factor and power distribution with southeast BP.

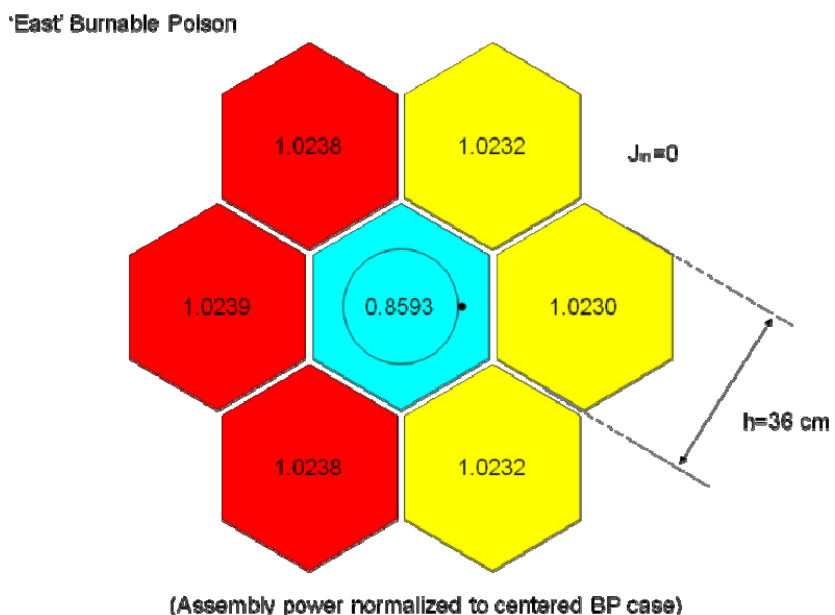

Figure 25. Simplified mini-core effective multiplication factor and power distribution with east BP.

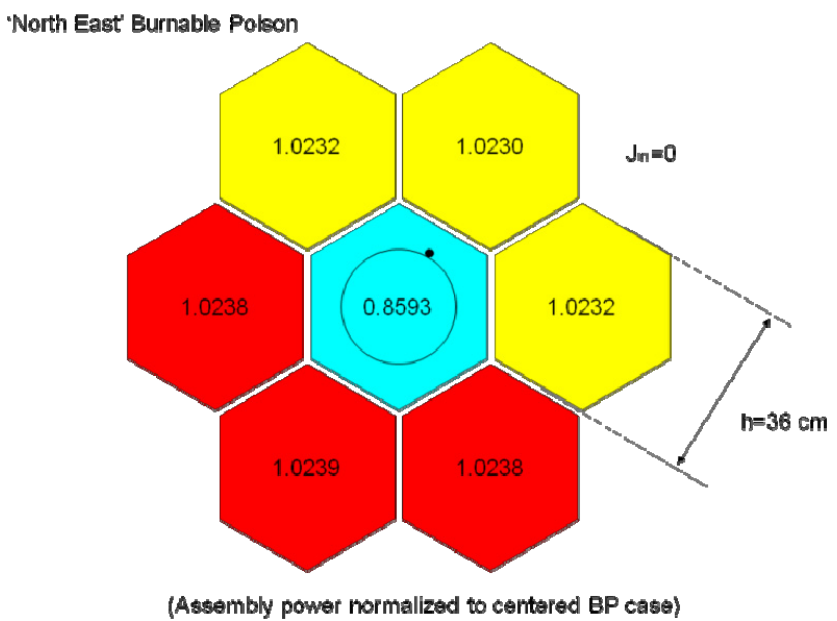

Figure 26. Simplified mini-core effective multiplication factor and power distribution with northeast BP. 


\section{CONCLUSIONS}

Two methods for introducing transport corrections into nodal calculations have been presented. One method is meant for the treatment of control rods in PBRs in cylindrical geometry through the use of embedded transport solutions within the nodal diffusion code. The second method introduces the explicit treatment of strong absorbers directly within the nodal solution scheme and relies on a novel, incomplete, homogenization method to produce compatible effective reaction rates for the BPs. Both new methods have been successfully demonstrated and shown to be effective at significantly reducing the error from the traditional nodal approach.

A new hybrid method has been developed for control rod treatment that accurately captures the angular effects of a control rod. This new hybrid method was embedded inside an R-Z and R-Theta diffusion solver and has been shown to produce errors that are acceptable for real reactor physics calculations and applications. The embedding method is seamless and very flexible because response nodes can be changed out as necessary, unlike previous methodologies. The implementation of the method in R-Z-theta has been initiated, with all diffusion theory side work completed (embedding framework coded). The testing of the R-Z-theta methodology is not possible this fiscal year because the transport solver to be embedded is not yet available. The reason for this is that development of the overall method was performed in collaboration with Georgia Tech, with Idaho National Laboratory responsible for the embedding and testing of the transport solver within the diffusion code and Georgia Tech responsible for producing the transport solvers to be embedded. The portion of work done by Idaho National Laboratory was supported primarily by the NGNP project. The work done by Georgia Tech was supported by a Nuclear Energy Research Initiative project that is not yet completed. The final transport solver will not be available until next fiscal year. At that point the testing of the 3-D implementation, that remains to be performed, should be completed.

This report outlines a novel treatment for BPs within the nodal method embodied in the HEXPEDITE code and presented the related "incomplete homogenization procedure." These developments were carried out under the assumption of neutron diffusion theory. Particularly, the Nodal Green's Function Method approach was adopted for solving the diffusion equation because it allows for the solution of problems involving complicated source shapes, beyond those that can be represented by low-order polynomials, which is especially warranted in this case because local intra-assembly BPs are modeled as delta distributions, thus requiring special treatment. The model of BPs as delta distributions is motivated by the physical observation that standard homogenization procedures tend to smear the effects of strong localized absorbers. The treatment of the BP as a delta distribution is constrained by imposing the conservation of the physical absorption reaction rate via the neutron balance equation. In order to generate the necessary data, traditional flux-weighted homogenization is generalized to create a separate, absorberspecific, cross-section.

The modified neutron diffusion model with a space-dependent removal cross-section is discretized via the Nodal Green's Function Method. While the BP adds extra complexity in the derivation of the 1-D transverse-averaged solution, it is relatively straightforward to implement and requires only an extra set of data.

Finally, numerical results were obtained for a set of manufactured problems for which the exact solution is known. These manufactured solutions were used to verify the implementation of the BP treatment into the HEXPEDITE code. Those results are not included in this report.

The development of the BP treatment methodology is complete, including the new incomplete homogenization scheme and the development of the theory for the modification of a nodal solver. The new homogenization method was tested using the HELIOS code. The theory for explicit treatment of BP was also completed, but its testing was limited to manufactured solutions (as stated above) and to numerical implementation on a small (toy) problem using limited ad-hoc modifications of the HEXPEDITE code. The implementation into a general full-production code has yet to proceed. 
The method for treating BP could be generalized as the treatment of control rods in prismatic HTRs. The embedded transport method that was demonstrated for cylindrical geometry could be generalized to the hexagonal geometry. Either (or possibly both) of these developments should be considered. 


\section{COMPUTER HARDWARE AND CODES}

The analysis of control rods in the pebble bed reactor was performed by modifying the CYNOD22 code. The code was executed on a Dell T5400 2.66 GHz quad core computer running Windows XP operating system (Service Pack 3). Cross section data sets are supplied by the user.

The analysis of burnable poisons in prismatic reactor fuel blocks was performed by modifying the HEXPEDITE19 code. The code was executed on a Dell Inspiron 64-bit Workstation running the LINUX SUSE 11.1 operating system. Cross section data sets are supplied by the user. 


\section{REFERENCES}

1. Scherer, W. and H. J. Neff, "Determination of Equivalent Cross Sections for Representation of Control Rod Regions in Diffusion Calculations," Jul 1311, 1976.

2. Fen, V., et al., "Modeling of Neutron Absorbers in High Temperature Reactors by Combined Transport-Diffusion Methods," Jul 2573, 1992.

3. Ougouag, A., et al., "Development of Method for Neutronics Treatment of Non-multiplying Zones in Pebble Bed Reactors", Proceedings HTR 2006: $3^{\text {rd }}$ International Topical Meeting on Hight Termperature Reactor Technology, Johannesburg, South Africa, October 1-4, 2006.

4. Zhang, D., et al., "A Hybrid Diffusion/Transport Method," Proceedings of the International Conference on Mathematics, Computational Methods \& Reactor Physics 2009, Saratoga Springs, New York, May 2009.

5. Duderstadt, J. L., and L. J. Hamilton, "Nuclear Reactor Analysis" John Wiley and sons, Inc., 1976

6. Ougouag, A. M., "A Coarse-Mesh Nodal Method for Multigroup Multidimensional Neutron Diffusion Computations," M.S. Thesis, University of Illinois, 1981.

7. Rajic, H. L., and A. M. Ougouag, "ILLICO,: A Nodal Neutron Diffusion Method for Modern Computer Architectures," Nuclear Science and Engineering, Vol. 103, Issue 4, 1989, pp. 392-408.

8. Smith, K. S., "Assembly homogenization techniques for light water reactors," Prog. Nucl. Energy, Vol. 17, 1986, p. 303.

9. Rahnema, F. and E. M. Nichita, "Leakage Corrected Spatial (Assembly) Homogenization Technique," Ann. Nucl. Energy, Vol. 24, No. 6, 1997, pp. 477-488.

10. Hoffman, J. Numerical Methods for Engineers and Scientist, New York, USA, McGraw Hill, 1992.

11. Henry, A. F., "Refinements in Accuracy of Coarse-Mesh Finite-Difference Solution of the Group Diffusion Equations," Numerical Reactor Calculations, Vienna, International Atomic Energy Agency, 1972.

12. Mosher, S. W, et al., "Monte Carlo Adaption of a Heterogeneous Course Mesh Transport Method," Trans. Am. Nucl. Soc., Vol. 89, 310, (2003)

13. Forget, B., et al, "A Heterogeneous Coarse Mesh Method for the 2-D NEA C5G7 MOX Benchmark Problem," Progress in Nuclear Energy, Vol. 45, Nos. 2-4, 2004, pp. 233-254.

14. Lawrence, R.D., "Progress in Nodal Methods for the Solution of the Neutron Diffusion and Transport Equations," Prog. Nucl. Energy, Vol. 17, No. 3, 1986, p. 271.

15. Koebke, K., 1978. “A New Approach to Homogenization and Group Condensation. ” IAEATECDOC-231, International Atomic Energy Agency, 1978.

16. Rajic, H. L., and A. M. Ougouag, "An Advanced Nodal Neutron Diffusion Method with SpaceDependent Cross Sections: ILLICO-VX,” Trans. ANS, Vol. 55, 1987, pp. 342-343.

17. Rajic, H. L., and A. M. Ougouag, "A Burnup Corrected 3-D Nodal Depletion Method for Vector and Parallel Computer Architecture", in The First International Conference on Supercomputing in Nuclear Application (SNA '90) proceedings of a conference held March 12-16, 1990, in Mito, Japan, pp.336-340, Japan Atomic Energy Research Institute, Nuclear Energy Data Center, 1990.

18. R. Sanchez, Assembly Homogenization Techniques for Core Calculations. Prog. Nucl. Energy, Vol. 51, 2009, pp. 14-31. 
19. Fitzpatrick, W. E., and A. M. Ougouag, "HEXPEDITE: A Net Current Multigroup Nodal Diffusion Method for Hexagonal-z Geometry," Trans. Am. Nucl. Soc., Vol. 66, 1992.

20. Ougouag, A. M., and W. Fitzpatrick, "An Inherently Parallel Multigroup Nodal Diffusion Method for Hexagonal-Z Geometry," Fourth International Conference on Simulation Methods in Nuclear Engineering, Montreal, Canada, June 2-4, 1993.

21. W. E. Fitzpatrick, "Developments in Nodal Reactor Analysis Tools for Hexagonal Geometry," Doctoral dissertation, University of Illinois at Urbana-Champaign, 1995.

22. Hiruta, H., Ougouag, A., Gougar, H., and Ortensi, J, "CYNOD: A Neutronics Code for Pebble Bed Modular Reactor Coupled Transient Analysis", INL/CON-08-14160, April 2008. 